\title{
1 Multiplex, translaminar imaging in the spinal cord of behaving mice
}

2 Pavel Shekhtmeyster ${ }^{1,2, \#, ~ E r i n ~ M . ~ C a r e y ~}{ }^{1, \#}$, Daniela Duarte ${ }^{1}$, Alexander Ngo ${ }^{1}$, Grace Gao,

3 Nicholas A. Nelson ${ }^{1,3}$, Charles L. Clark ${ }^{1}$, and Axel Nimmerjahn ${ }^{1,{ }^{\star}}$

$4 \quad{ }^{1}$ Waitt Advanced Biophotonics Center, The Salk Institute for Biological Studies, La Jolla, CA

5 92037, USA; ${ }^{2}$ Electrical and Computer Engineering Graduate Program, University of California,

6 San Diego, La Jolla, CA 92037, USA; ${ }^{3}$ Biological Sciences Graduate Program, University of

7 California, San Diego, La Jolla, CA 92037, USA

$8 \quad$ \#These authors contributed equally to this work.

$9 \quad{ }^{*}$ Correspondence: animmerj@salk.edu (A.N.)

\section{Abstract}

11 While the spinal cord is known to play critical roles in sensorimotor processing, including pain-

12 related signaling, corresponding activity patterns in genetically defined cell types across spinal

13 laminae have remained elusive. Calcium imaging has enabled cellular activity measurements in

14 behaving rodents but is currently limited to superficial regions. Using chronically implanted

15 microprisms, we imaged sensory and motor evoked activity in regions and at speeds

16 inaccessible by other high-resolution imaging techniques. To enable translaminar imaging in

17 freely behaving animals through implanted microprisms, we additionally developed wearable

18 microscopes with custom-compound microlenses. This new integrated system addresses

19 multiple challenges of previous wearable microscopes, including their limited working distance,

20 resolution, contrast, and achromatic range. The combination of these innovations allowed us to

21 uncover that dorsal horn astrocytes in behaving mice show somatosensory program-dependent

22 and lamina-specific calcium excitation. Additionally, we show that tachykinin precursor 1 (Tac1)-

23 expressing neurons exhibit upper laminae-restricted activity to acute mechanical pain but not

24 locomotion. 


\section{Introduction}

26 The spinal cord plays crucial roles in somatosensation ${ }^{1-3}$. Genetic, pharmacologic, anatomical,

27 and electrophysiological studies have revealed that specific somatosensory programs, such as

28 pain or movement, are linked to spatially restricted modules comprised of heterogeneous

29 neuronal cell types. These modules reside in defined laminae, located at different depths from

30 the spinal surface ${ }^{1-3}$. However, genetic and pharmacologic approaches lack the spatial and

31 temporal resolution to investigate cellular activity patterns that underlie somatosensory

32 computations and typically rely on overt behavioral phenotypes to define cell types' functional

33 roles. Histological methods can identify highly active neurons across spinal laminae (e.g., cFos

34 expression) but generate only snapshots at discrete time points. Electrophysiology approaches

35 are valuable for probing neuronal activity, but recordings in behaving animals from genetically

36 defined cells across spinal laminae remain to be demonstrated. Emerging evidence also

37 suggests that spinal astrocytes respond to different kinds of neural activity with calcium

38 excitation and modulate pain signaling and motor function ${ }^{4-8}$. Astrocyte activity remains difficult

39 to interrogate with the methods mentioned above.

Fluorescence imaging has recently allowed real-time measurement of neuronal and astrocyte activity in the spinal cord of behaving mice ${ }^{4}$. However, scattering and absorption restrict optical access to superficial dorsal horn regions. This is because the spinal gray matter is surrounded by thick myelin layers that are highly reflective across a wide wavelength range. This anatomical arrangement limits one- and two-photon imaging to about 75-150 $\mu \mathrm{m}$ and 150-350 $\mu \mathrm{m}$ depths, respectively, depending on the fluorescence indicator employed ${ }^{9}$. With three-photon

47 microscopy, depths of about 350-500 $\mu \mathrm{m}$ can be achieved, but at the expense of acquisition

48 frame rate, the field of view (FOV), or multi-color imaging capabilities ${ }^{10}$. Low acquisition frame

49 rates are particularly detrimental to spinal cord recordings, as they hamper computational

50 correction of tissue movement-induced image artifacts. The large-amplitude and non-uniform 
51 tissue displacements typical during animal behavior are also the reason why

52 electrophysiological recordings regularly used in other central nervous system regions have

53 proven extremely challenging in the spinal $\operatorname{cord}^{9}$.

55 The implantation of micro-optics, such as gradient-index (GRIN) lenses, has allowed access to

56 deep tissue regions in the brain. However, typical GRIN lens diameters $(\geq 0.5 \mathrm{~mm})$ are

57 comparable to the spinal gray matter's width in mice, preventing their insertion without undue

58 tissue disruption. In contrast, glass reflective microprisms have enabled optical access to brain

59 areas difficult if not impossible to reach otherwise, such as prefrontal and entorhinal cortex, and

60 without tissue removal ${ }^{11-13}$. Whether and how a microprism-based approach could be applied to

61 the spinal cord remains unclear.

63 Imaging through implanted microprisms requires long working distance (WD) objectives. Current

64 wearable microscopes for imaging in freely behaving animals do not offer WDs $>1 \mathrm{~mm}$ and

65 often rely on GRIN objectives, preventing high-resolution imaging through these micro-

66 optics $^{9,14,15}$. The reliance on GRIN-based imaging optics also limits multi-color recording

67 capabilities due to their narrow achromatic range (tens of nanometers) ${ }^{16}$.

69 These technical barriers in both surgery and imaging have prevented measurement of cellular

70 activity in deep sensory and premotor areas and at high speed across spinal laminae of

71 behaving animals, limiting our ability to uncover how the spatiotemporal activity patterns in

72 genetically defined cell types contribute to sensorimotor processing. To address these long-

73 standing challenges, we introduce several innovations, including 1) a wearable microscope with

74 custom-compound-microlenses allowing high-speed (45 fps), high-resolution and high-contrast

$75(\sim 1.5 \mu \mathrm{m})$, and multi-color imaging (500-620 nm spectral range) through tissue-implanted micro- 
optics (2.4-mm working distance), and 2) a surgical procedure that enables long-term activity

77 measurements across spinal laminae using implanted microprisms. Using these methods, we

78 show that tachykinin precursor 1 (Tac1)-expressing neurons respond rapidly to painful

79 mechanical stimuli specifically in sensory dorsal horn laminae. In contrast, astrocytes showed

80 slow calcium transients to the same stimuli in sensory and motor-evoked activity in premotor

81 areas, suggesting region-dependent (i.e., lamina- and neuronal cell type-dependent) functional

82 signaling within this glial cell population.

\section{Results}

85 Wearable microscopes with long working distance custom-compound-microlenses for

86 high-resolution, high-contrast measurements in freely behaving mice. To meet the

87 application needs mentioned above, as well as match or exceed the optical performance

88 characteristics of comparable wearable devices, the design goals for our microscope's optical

89 system included a long working distance $(>2 \mathrm{~mm})$, high spatial resolution $(1-2 \mu \mathrm{m}$ across the

90 field of view), high contrast (MTF10 < $1.5 \mu \mathrm{m})$, and a broad achromatic range $(450-650 \mathrm{~nm})$.

91 Additional design parameters included an >0.4 numerical aperture, $5 x$ magnification

92 (corresponding to an $\sim 900 \mu \mathrm{m} \times 700 \mu \mathrm{m}$ field of view), and $20 \mathrm{~mm}$ track length.

94 Our optical design included six custom microlenses to meet these goals (Fig. 1a; Suppl. Table

95 1). The lens surface profile, radius of curvature, distance between surfaces, and glass types

96 were optimized using Zemax modeling. A \#0 coverslip ( 100 $\mu$ m thickness) was included in the

97 design to minimize optical aberrations when imaging through live animal-implanted glass

98 windows. Additionally, we accounted for a filter cube required for fluorescence imaging, placed

99 in the collimated space between the lenses. Following optical modeling, the lenses and brass

100 spacers were fabricated and assembled into two custom-made barrel holders minimized in size 
and weight (Fig. 1b-c). Each assembled barrel weighed $<0.2 \mathrm{~g}$.

Next, we characterized the performance of the custom microlens system. Based on our Zemax calculations, the predicted lateral and axial point spread function (PSF) in the center of the field of view (FOV) was $0.56 \mu \mathrm{m}$ and $5.16 \mu \mathrm{m}$, respectively. Experimentally, we measured $0.68 \pm$ $0.01 \mu \mathrm{m}$ and $7.85 \pm 0.33 \mu \mathrm{m}$ (Suppl. Fig. 1; Fig. 3), indicating that lens fabrication and assembly only introduced minor errors. These measurements used a glass blank of similar thickness and composition in lieu of the filter cube, a separate potential source of aberrations.

109 Our interchangeable filter cube consisted of multi-band excitation and emission filters, a custom 110 multi-band dichroic beamsplitter, and two fused silica microprisms, joined together using an 111 index-matching optical adhesive. This all-glass assembly minimized refractive index mismatch 112 at the dichroic's interfaces, preventing lateral beam offset and associated aberrations. A flexible 113 PMMA fiber was used to deliver excitation light (Fig. 1a, c), providing flexibility in multi-color imaging applications. This design offers several advantages: a) It allows simultaneous coupling of multiple high-power light sources, such as DPSS lasers, enabling efficient excitation of coexpressed fluorophores (or opsins); and b) it provides flexibility in selecting functional reporters

117 (and actuators).

To match the optical system's high resolution, we chose a CMOS sensor with a $3.75 \mu \mathrm{m}$ pixel spacing, corresponding to $0.69 \mu \mathrm{m}$ at the object plane. This sensor, available in both RGB and monochrome versions, offered high-sensitivity (5.48 and 6.7 V/lux-sec for the RGB and monochrome version, respectively) and frame rates (45 fps at $1280 \times 960$-pixel resolution) and was mounted on a custom miniature printed circuit board (PCB) (Methods). Using a high-

124 resolution microscopy target with spatial frequencies up to $3,300 \mathrm{lp} / \mathrm{mm}$, we found that our 125 integrated device had a limit of resolution (LOR) of $0.65 \pm 0.07 \mu \mathrm{m}$ and $0.84 \pm 0.28 \mu \mathrm{m}$ in the 126 center and at the edges of the FOV, respectively (Fig. 1d-e). Using a regularly spaced grid 
127 target with $100 \mu \mathrm{m}$ spacing, the FOV of our integrated system was $911 \mu \mathrm{m} \times 703 \mu \mathrm{m}$.

129 Next, to determine our microscope's contrast limit, we measured the modulation transfer

130 function (MTF) using the Slanted Edge test and MTF10 metric (i.e., contrast at 10\%). Although

131 MTF is frequently reported at $0 \%$, in our experience, MTF values below $10 \%$ are affected by

132 sensor noise limits and optical phase reversal. The MTF10 of our integrated device was 303

$133 \mathrm{Ip} / \mathrm{mm}$ tangential $(1.65 \mu \mathrm{m}$ line width) and $373 \mathrm{Ip} / \mathrm{mm}$ sagittal $(1.34 \mu \mathrm{m}$ line width) in the center

134 of the FOV (Suppl. Fig. 2).

136 Our microscope's working distance (WD) was determined to be $2.365 \mathrm{~mm}$ (from the edge of the 137 objective barrel to the microscopy target in air) using a precision differential actuator and grid

138 target. This exceptionally long WD enables imaging through implantable micro-optics, such as

139 microprisms (see below). Our fully assembled device measured 7 x $14 \times 20 \mathrm{~mm}$ and weighed

1402.96 grams, making it suitable for applications in mice. Together, these data demonstrate that

141 our custom-compound-microlens system simultaneously provides exceptional spatial resolution,

142 contrast, and working distance while retaining the large field of view, small form factor, and low

143 weight compared to existing wearable microscopes.

145 Multiplex imaging in behaving mice. Sensorimotor processing involves the dynamic interplay

146 of numerous cell types' activity. To evaluate our integrated system's ability to perform multiplex

147 measurements, we first imaged a monolayer of cell body-sized $(15 \mu \mathrm{m})$ fluorescent micro-

148 particles emitting three different spectral bands. As shown in Fig. 2a-c, the various colored

149 microspheres could be brought into focus simultaneously, demonstrating the optical system's

150 low axial chromatic aberration across the FOV. To confirm this feature in a densely labeled

151 environment, we imaged spinal tissue sections with neurons and astrocytes stained in two 
colors (Fig. 2d). The stained cells and their processes were readily distinguishable, further demonstrating our wearable device's high resolution and contrast. As an additional test, we conducted multi-color imaging in behaving animals. GFAP-GCaMP6f mice, which constitutively express the green fluorescent calcium indicator GCaMP6f in astrocytes, were injected into the L3-L5 lumbar spinal cord with an AAV9-CaMKII-H2B-GCaMP7f-TagRFP vector, resulting in concurrent nuclear-localized GCaMP7f and TagRFP expression in excitatory neurons. Two to three weeks after the injection, we recorded noxious mechanical stimulus (tail pinch) evoked calcium activity in the superficial dorsal horn. To extract neurons' and astrocytes' evoked activity with minimal signal crosstalk (e.g., from out-of-focus cells), we defined computational exclusion criteria (e.g., TagRFP intensity level) to select appropriate regions of interest (ROls) (Fig. 2e-g)

(Methods). Consistent with our previous work ${ }^{4,9}$, astrocytes showed widespread calcium activity to noxious tail pinch $(p>500 \mathrm{~g}$ ) across the FOV, while running alone did not evoke concerted excitation in the superficial dorsal horn. In contrast, calcium transients in excitatory neuron nuclei were sparse ${ }^{17}$ and had lower signal amplitude. Their onset latencies and durations were comparable to astrocytes (Fig. $\mathbf{2 g}$ ).

Together, these data demonstrate that our custom-compound-microlens system and integrated microscopes allow simultaneous imaging of at least three colors, including calcium activity in different genetically defined cell populations of behaving mice, all with a single image sensor.

\section{Chronic translaminar imaging in the spinal cord of behaving mice through implanted}

173 microprisms. While wearable microscopes have uncovered sensory-evoked activity in the

174 superficial dorsal horn of awake mice ${ }^{4}$, sensory activity in the spinal cord is not limited to these 175 superficial regions. Measuring cellular activity in deeper and simultaneously across laminae at 176 high speeds requires devices with sufficient WD to image through implanted micro-optics. To 
177 test our integrated system's ability to enable such measurements, we first constructed tissue

178 analogs in which reflective glass microprisms of different sizes were embedded (Fig. 3a-c)

179 (Methods). These tissue phantoms also allowed us to quantify signal attenuation, imaging

180 depth, and depth-dependent resolution in a scattering medium (Fig. 3d-o). Embedded $6 \mu \mathrm{m}$

181 fluorescent beads were readily visible up to $\sim 350 \mu \mathrm{m}$ distance from the vertical microprism face

182 when imaging through a $0.7 \mathrm{~mm} \times 0.7 \mathrm{~mm} \times 0.7 \mathrm{~mm}(\mathrm{~W} \times \mathrm{D} \times \mathrm{H})$ (Fig. 3b, e, h, $\mathbf{k}, \mathbf{n})$ or custom

$183 \quad 0.7 \mathrm{~mm} \times 0.7 \mathrm{~mm} \times 1.7 \mathrm{~mm}$ microprism (Fig. 3c, f, i, I, o).

185 Next, we developed a surgical approach for microprism-assisted high-speed imaging across

186 spinal laminae in vivo, including regions inaccessible by other high-resolution techniques. We

187 found that inserting a $0.7 \mathrm{~mm} \times 0.7 \mathrm{~mm} \times 0.7 \mathrm{~mm}$ microprism at the lateral interface between the

188 spinal gray and white matter was associated with minimal loss of NeuN-positive neurons two to

189 four weeks after implantation (Fig. 4a; Suppl. Fig. 3). Microglia and astrocyte inflammatory

190 responses were transient and confined to regions near the microprism-tissue interface, steadily

191 declining over the four-week implantation period (Suppl. Figs. 4-5). Microprism-implanted mice

192 did not show overt signs of sensory or motor deficits, as quantified by von Frey and kinematic

193 weight-bearing tests (Suppl. Fig. 6). To determine whether our approach allows translaminar

194 activity measurements in behaving animals, we performed optical recordings with our new

195 wearable microscopes in the lumbar spinal cord of calcium indicator-expressing transgenic

196 mice. Optical recordings were performed at $75 \mu \mathrm{m}$ and $125 \mu \mathrm{m}$ distance from the vertical prism

197 face (Fig. 4a-c). Given these recording positions and the spinal cord's known functional laminar

198 arrangement, sensory stimuli and motor-evoked cellular activity would be expected to manifest

199 in central and lower FOV regions, respectively, provided the investigated cells process

200 corresponding sensorimotor information (Fig. 4a-b; Suppl. Fig. 3). To comprehensively quantify

201 the spatial and temporal properties of cells' activity patterns, we tiled the FOV with equally sized 
$202(10 \mu \mathrm{m} \times 10 \mu \mathrm{m}) \mathrm{ROIs}$, excluding blood vessel regions (Methods). We chose noxious tail pinch

$203(p>500 \mathrm{~g})$ as sensory stimuli for comparison with our previous work ${ }^{4}$. Starting with GFAP-

204 GCaMP6f mice ( $N=5)$, we found that astrocytes exhibit calcium excitation across $\sim 200 \mu$ m-wide

205 central FOV regions (Fig. 4d-f; Suppl. Movie 1), corresponding to sensory laminae. This areal

206 activation was remarkably robust across animals, suggesting high reproducibility of our surgical

207 preparation (Fig. 4f). Population transients evoked by noxious pinch had a $3.34 \pm 0.21$ s onset

208 latency (Fig. 4g, left) and $2.79 \pm 0.09$ s duration (Fig. 4h, right), whereas individual ROI

209 transients lasted $2.2 \pm 0.02 \mathrm{~s}$ (Fig. 4h, left). In contrast, running-evoked astrocyte excitation in

210 the same mice occurred in $~ 300 \mu \mathrm{m}$-wide lower FOV regions (Fig. 4i-k; Suppl. Movie 2), likely

211 corresponding to premotor areas. Average transients evoked by 2-4 s-long runs had 3.39 \pm 0.19

212 s onset latency (Fig. $\mathbf{4 g}$, right) and $2.75 \pm 0.12$ s duration (Fig. 4I, right), while individual ROI

213 transients lasted $2.53 \pm 0.03 \mathrm{~s}$ (Fig. 4l, left). Not every run triggered an astrocyte response,

214 particularly after short inter-run rest periods (Fig. 4j), an effect previously described for motor-

215 related brain areas $^{18,19}$.

217 Next, we performed imaging in Tac1-GCaMP6f mice $(\mathrm{N}=3)$ with calcium indicator expression in

218 Tac1-expressing neurons involved in pain processing ${ }^{20,21}$. Noxious tail pinch $(p>500 \mathrm{~g})$ evoked

219 calcium excitation across $\sim 300 \mu \mathrm{m}$-wide central FOV regions (Fig. 5a-c; Suppl. Movie 3).

220 Average transients across ROls had an $0.54 \pm 0.06 \mathrm{~s}$ onset latency (Fig. 5d) and $3.29 \pm 0.23 \mathrm{~s}$

221 duration (Fig. 5e, right), whereas individual ROI transients lasted $2.20 \pm 0.02$ s (Fig. 5e, left),

222 indicating signal propagation within and across laminae (Fig. 5b). Notably, unlike astrocytes,

223 Tac1-expressing neurons did not show running evoked activity in premotor FOV areas (Fig. 5f-

224 h). 
226 Together, these data demonstrate that our approach provides real-time visualization of how

227 different somatosensory programs activate different cell types in spatially restricted dorsoventral

228 regions of behaving mice. Astrocytes showed acute mechanical pain and motor evoked calcium

229 excitation in superficial and deep laminae, respectively. Tac1-expressing neurons responded to

230 acute mechanical pain with upper but not deep lamina activation. These neurons also did not

231 respond to locomotion, making them unlikely contributors to astrocytes' motor evoked

232 responses.

\section{Discussion}

235 In this study, we have developed methods for high-speed translaminar activity measurements

236 from genetically defined cell types in the spinal cord of behaving mice. Using chronically

237 implanted micro-prisms with a $700 \mu \mathrm{m}$ FOV, we could image sensory and motor evoked activity

238 in sensory and premotor regions, respectively (Figs. 4-5). Our histological, behavioral, and

239 functional data indicate that spinal microprism implantation is remarkably well tolerated by adult

240 mice (Suppl. Figs. 3-6). To enable translaminar imaging in freely behaving animals, we

241 developed and thoroughly characterized wearable microscopes with custom-compound

242 microlenses (Figs. 1-3; Suppl. Figs. 1-2). This new integrated system offers a 2.4 mm working

243 distance, $\sim 1.5 \mu \mathrm{m}$ lateral resolution and MTF10 contrast, and 500-620 nm achromatic range,

244 thereby overcoming multiple challenges of previous wearable microscopes. The combination of

245 these innovations allowed us to uncover that dorsal horn astrocytes in behaving mice show

246 distinct somatosensory program-dependent and lamina-specific calcium excitation (Fig. 4).

247 Additionally, we found that Tac1-expressing neurons exhibit upper laminae-restricted activity to

248 acute mechanical pain but not locomotion (Fig. 5).

250 Most previous wearable one-photon microscopes, including our own ${ }^{4,22,23}$, rely on gradient-index 
251 lenses ${ }^{9,14,15}$. While the optical capabilities of these lenses have seen significant progress,

252 performance limitations remain, such as their narrow achromatic range, short working distance,

253 or variable resolution and contrast across the field of view ${ }^{16}$. Wearable one-photon microscopes

254 with more traditional lens systems have emerged but tend to use few or off-the-shelf optical

255 elements ${ }^{9,14,15}$, likely because of the difficulty of modeling, optimizing, or producing more

256 complex compound-microlens systems, thereby constraining the number of desirable optical

257 features that can be achieved concurrently. We, therefore, took an ab initio approach that

258 involved paraxial calculations and Zemax modeling (Methods). Our custom-compound-

259 microlens system provides a wide achromatic range (from the visible to near-infrared), high

260 spatial resolution and contrast, and an exceptionally long working distance (Figs. 1-3; Suppl.

261 Figs. 1-2).

262

263 Partly because of these technical limitations, previous imaging approaches could not record

264 cellular activity across sensory and premotor areas, preventing vital biological questions from

265 being addressed ${ }^{9}$. For example, recent gene expression and electrophysiological studies have

266 indicated that astrocytes show lamina-specific properties ${ }^{7,24,25}$, raising questions about how this

267 heterogeneity might be linked to differences in functional signaling. Our data show that acute

268 pain and animal locomotor activity evoke astrocyte calcium excitation in distinct dorsal horn

269 regions (Fig. 4), supporting astrocytes' responsiveness and functional adaptation to region-

270 specific neural circuits and signaling. Likewise, genetic, immunohistological, and

271 electrophysiological approaches have implicated Tac1-expressing neurons in pain signaling ${ }^{20,21}$.

272 Our data show that noxious mechanical stimuli lead to rapid and widespread calcium activity

273 across dorsal horn laminae in these neurons (Fig. 5), the first such measurements in behaving

274 animals. 
276 High-speed translaminar imaging was achieved by microprism implantation. Developing this

277 approach for the spinal cord was a non-trivial endeavor given its small cross-sectional

278 dimensions compared to the brain, the limited space within mouse vertebrae, and region-

279 dependent tissue displacements during animal behavior ${ }^{4,26}$. We found that $0.7 \mathrm{~mm} \times 0.7 \mathrm{~mm} \times$

$280 \quad 0.7 \mathrm{~mm}$ microprisms provide an acceptable FOV while minimizing tissue disruption. No

281 significant loss of neuronal cell bodies was observed 2-4 weeks after implanting microprisms at

282 the lateral interface between the spinal gray and white matter (Suppl. Fig. 3). Glial reactivity

283 was transient and confined to regions near the tissue-microprism interface (Suppl. Figs. 4-5).

284 Sensory and motor tests before and after microprism implantation also revealed no overt

285 behavioral deficits (Suppl. Fig. 6), and our calcium imaging data suggest that spinal neurons

286 and astrocytes remain responsive to sensory input and motor actions (Figs. 4-5). Astrocyte

287 response properties to noxious tail pinch (e.g., onset latency, duration) were comparable to data

288 from non-implanted mice ${ }^{4}$. Nevertheless, microprism implantation causes some circuit disruption

289 (e.g., to white matter tracts near the implantation site) and should be considered in future

290 biological applications on a case-by-case basis, as is generally advisable for any imaging

291 approach that uses implanted micro-optics.

293 Repeated imaging through spinal cord-implanted microprism is feasible over at least four

294 weeks. This recording period may enable the study of prolonged structural and functional

295 biological processes, such as disease- or treatment-related dynamics (e.g., amyotrophic lateral

296 or multiple sclerosis progression, transplanted neural stem cell integration) $)^{9,10,27}$. Optical

297 recordings from the spinal ventral horn might be feasible with custom micro-optics. As

298 demonstrated in Fig. 3, our wearable microscopes have sufficiently long WD to image through

$2990.7 \mathrm{~mm}$ microprisms with a $1 \mathrm{~mm}$ vertical extension, potentially allowing the study of motor

300 neuron activity in relation to limb movement. Our surgical and optical methods may also apply to 
301 thoracic and cervical spinal cord regions, allowing the study of sensorimotor activity related to

302 internal organ or forelimb function. However, this will likely require the development of imaging

303 chambers specifically adapted to the different vertebra anatomy in these regions. Furthermore,

304 given the rapidly expanding number, color palette, and signal-to-noise ratio of genetically

305 encoded indicators (e.g., for neurotransmitters and neuromodulators) ${ }^{28}$, our wearable

306 microscopes' multiplex imaging capability might in the future enable the study of input-output

307 relationships (e.g., integration of molecular signals by a given cell type or modulation of local

308 cellular activity by descending projection fibers from the brain). In summary, our novel surgical

309 and optical techniques should enable a wide range of biological interrogations, providing

310 unprecedented opportunities for elucidating cellular and molecular mechanisms of sensorimotor

311 information processing in the spinal cord of behaving animals. 


\section{References}

3141 Koch, S. C., Acton, D. \& Goulding, M. Spinal circuits for touch, pain, and itch. Annu Rev

315 Physiol 80, 189-217, doi:10.1146/annurev-physiol-022516-034303 (2018).

3162 Abraira, V. E. \& Ginty, D. D. The sensory neurons of touch. Neuron 79, 618-639,

317 doi:10.1016/j.neuron.2013.07.051 (2013).

3183 Peirs, C. \& Seal, R. P. Neural circuits for pain: Recent advances and current views.

319 Science 354, 578-584, doi:10.1126/science.aaf8933 (2016).

3204 Sekiguchi, K. J. et al. Imaging large-scale cellular activity in spinal cord of freely

321 behaving mice. Nat Commun 7, 11450, doi:10.1038/ncomms11450 (2016).

3225 Kronschlager, M. T. et al. Gliogenic LTP spreads widely in nociceptive pathways.

323 Science 354, 1144-1148, doi:10.1126/science.aah5715 (2016).

3246 Kelley, K. W. et al. Kir4.1-dependent astrocyte-fast motor neuron interactions are

325 required for peak strength. Neuron 98, 306-319 e307, doi:10.1016/j.neuron.2018.03.010

326 (2018).

3277 Kohro, Y. et al. Spinal astrocytes in superficial laminae gate brainstem descending

328 control of mechanosensory hypersensitivity. Nat Neurosci 23, 1376-1387,

329 doi:10.1038/s41593-020-00713-4 (2020).

$3308 \mathrm{Xu}, \mathrm{Q}$. et al. Astrocytes contribute to pain gating in the spinal cord. Sci Adv 7, eabi6287, 331 doi:10.1126/sciadv.abi6287 (2021).

3329 Nelson, N. A., Wang, X., Cook, D., Carey, E. M. \& Nimmerjahn, A. Imaging spinal cord 333 activity in behaving animals. Exp Neurol 320, 112974,

334 doi:10.1016/j.expneurol.2019.112974 (2019).

33510 Cheng, Y. T., Lett, K. M. \& Schaffer, C. B. Surgical preparations, labeling strategies, and 336 optical techniques for cell-resolved, in vivo imaging in the mouse spinal cord. Exp Neurol 337 318, 192-204, doi:10.1016/j.expneurol.2019.05.010 (2019). 
33811 Andermann, M. L. et al. Chronic cellular imaging of entire cortical columns in awake 339 mice using microprisms. Neuron 80, 900-913, doi:10.1016/j.neuron.2013.07.052 (2013).

34012 Low, R. J., Gu, Y. \& Tank, D. W. Cellular resolution optical access to brain regions in 341 fissures: imaging medial prefrontal cortex and grid cells in entorhinal cortex. Proc Natl $342 \quad$ Acad Sci U S A 111, 18739-18744, doi:10.1073/pnas.1421753111 (2014).

34313 Heys, J. G., Rangarajan, K. V. \& Dombeck, D. A. The functional micro-organization of 344 grid cells revealed by cellular-resolution imaging. Neuron 84, 1079-1090, 345 doi:10.1016/j.neuron.2014.10.048 (2014).

34614 Aharoni, D. \& Hoogland, T. M. Circuit investigations with open-source miniaturized 347 microscopes: Past, present and future. Front Cell Neurosci 13, 141, 348 doi:10.3389/fncel.2019.00141 (2019).

34915 Chen, S. et al. Miniature fluorescence microscopy for imaging brain activity in freely350 behaving animals. Neurosci Bull 36, 1182-1190, doi:10.1007/s12264-020-00561-z $351 \quad$ (2020).

35216 Matz, G., Messerschmidt, B. \& Gross, H. Design and evaluation of new color-corrected 353 rigid endomicroscopic high NA GRIN-objectives with a sub-micron resolution and large 354 field of view. Opt Express 24, 10987-11001, doi:10.1364/OE.24.010987 (2016).

35517 Simonetti, M. et al. Nuclear calcium signaling in spinal neurons drives a genomic 356 program required for persistent inflammatory pain. Neuron $77,43-57$, 357 doi:10.1016/j.neuron.2012.10.037 (2013).

35818 Nimmerjahn, A., Mukamel, E. A. \& Schnitzer, M. J. Motor behavior activates Bergmann 359 glial networks. Neuron 62, 400-412, doi:10.1016/j.neuron.2009.03.019 (2009).

36019 Merten, K., Folk, R. W., Duarte, D. \& Nimmerjahn, A. Astrocytes encode complex 361 behaviorally relevant information. bioRxiv, doi:

$362 \quad$ https://doi.org/10.1101/2021.1110.1109.463784 (2021). 
36320 Polgar, E. et al. Substance P-expressing neurons in the superficial dorsal horn of the 364 mouse spinal cord: Insights into their functions and their roles in synaptic circuits.

$365 \quad$ Neuroscience 450, 113-125, doi:10.1016/j.neuroscience.2020.06.038 (2020).

$36621 \quad$ Huang, T. et al. Identifying the pathways required for coping behaviours associated with 367 sustained pain. Nature 565, 86-90, doi:10.1038/s41586-018-0793-8 (2019).

36822 Flusberg, B. A. et al. High-speed, miniaturized fluorescence microscopy in freely moving 369 mice. Nat Methods 5, 935-938, doi:10.1038/nmeth.1256 (2008).

37023 Ghosh, K. K. et al. Miniaturized integration of a fluorescence microscope. Nat Methods $371 \quad$ 8, 871-878, doi:10.1038/nmeth.1694 (2011).

$37224 \quad$ Kronschlager, M. T. et al. Lamina-specific properties of spinal astrocytes. Glia 69, 1749$373 \quad$ 1766, doi:10.1002/glia.23990 (2021).

37425 Molofsky, A. V. et al. Astrocyte-encoded positional cues maintain sensorimotor circuit 375 integrity. Nature 509, 189-194, doi:10.1038/nature13161 (2014).

37626 Harrison, M. et al. Vertebral landmarks for the identification of spinal cord segments in 377 the mouse. Neuroimage 68, 22-29, doi:10.1016/j.neuroimage.2012.11.048 (2013).

37827 Ceto, S., Sekiguchi, K. J., Takashima, Y., Nimmerjahn, A. \& Tuszynski, M. H. Neural 379 stem cell grafts form extensive synaptic networks that integrate with host circuits after 380 spinal cord injury. Cell Stem Cell 27, 430-440 e435, doi:10.1016/j.stem.2020.07.007 $381 \quad$ (2020).

38228 Sabatini, B. L. \& Tian, L. Imaging neurotransmitter and neuromodulator dynamics in vivo 383 with genetically encoded indicators. Neuron 108, 17-32, 384 doi:10.1016/j.neuron.2020.09.036 (2020). 

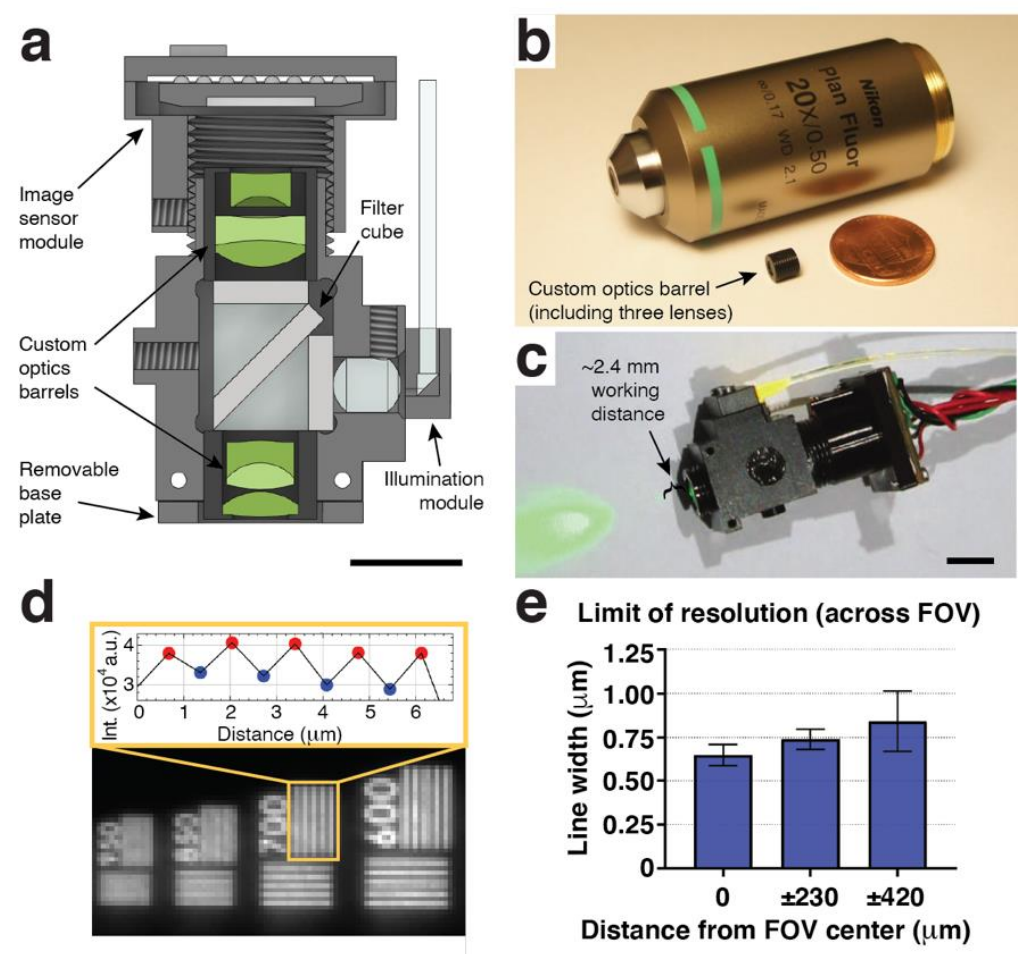

Fig. 1 | Wearable microscopes with long working distance custom-compound-

Cross-section of the wearable microscope and its custom compound micro-optics (green) for high-resolution, multi-color, and long working distance imaging. Scale bar, $5 \mathrm{~mm}$. b, Image of the $<0.2$-grams objective barrel including three miniature lenses next to a regular microscope objective and one-cent coin. c, Image of the fully assembled device with $~ 2.4-\mathrm{mm}$ working distance for high-resolution imaging through intermediary optics. Scale bar, $5 \mathrm{~mm}$. d, Bottom, image detail of a high-resolution microscopy target demonstrating the wearable microscope's resolving power. Top, intensity values of peaks and troughs across the indicated $700 \mathrm{lp} / \mathrm{mm}$ bar set. e, Limit of resolution across the field of view (FOV). Displayed values are averages across

398 the horizontal and vertical line target results from comparable positions to the left and right of 399 the FOV center. Spatial frequencies were converted to line widths. The data are presented as 400 mean \pm s.e.m. 

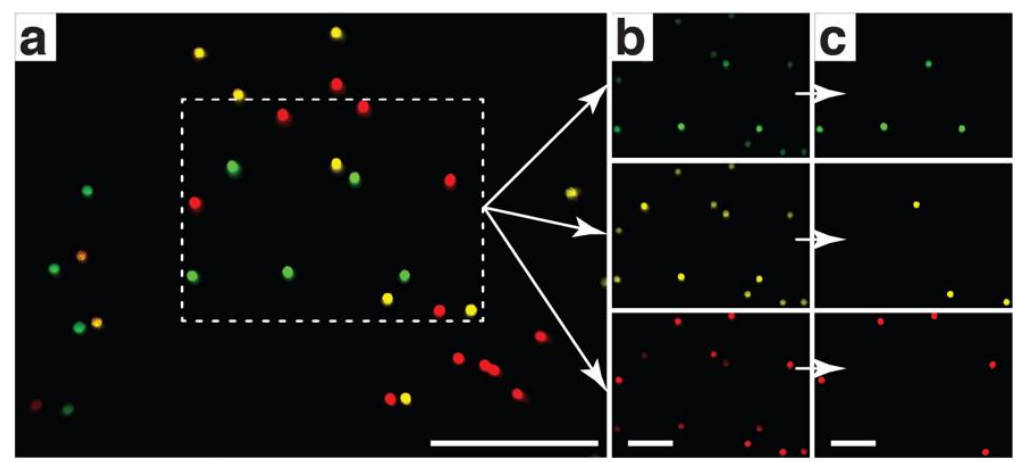

d Spinal dorsal horh (coronal slice)
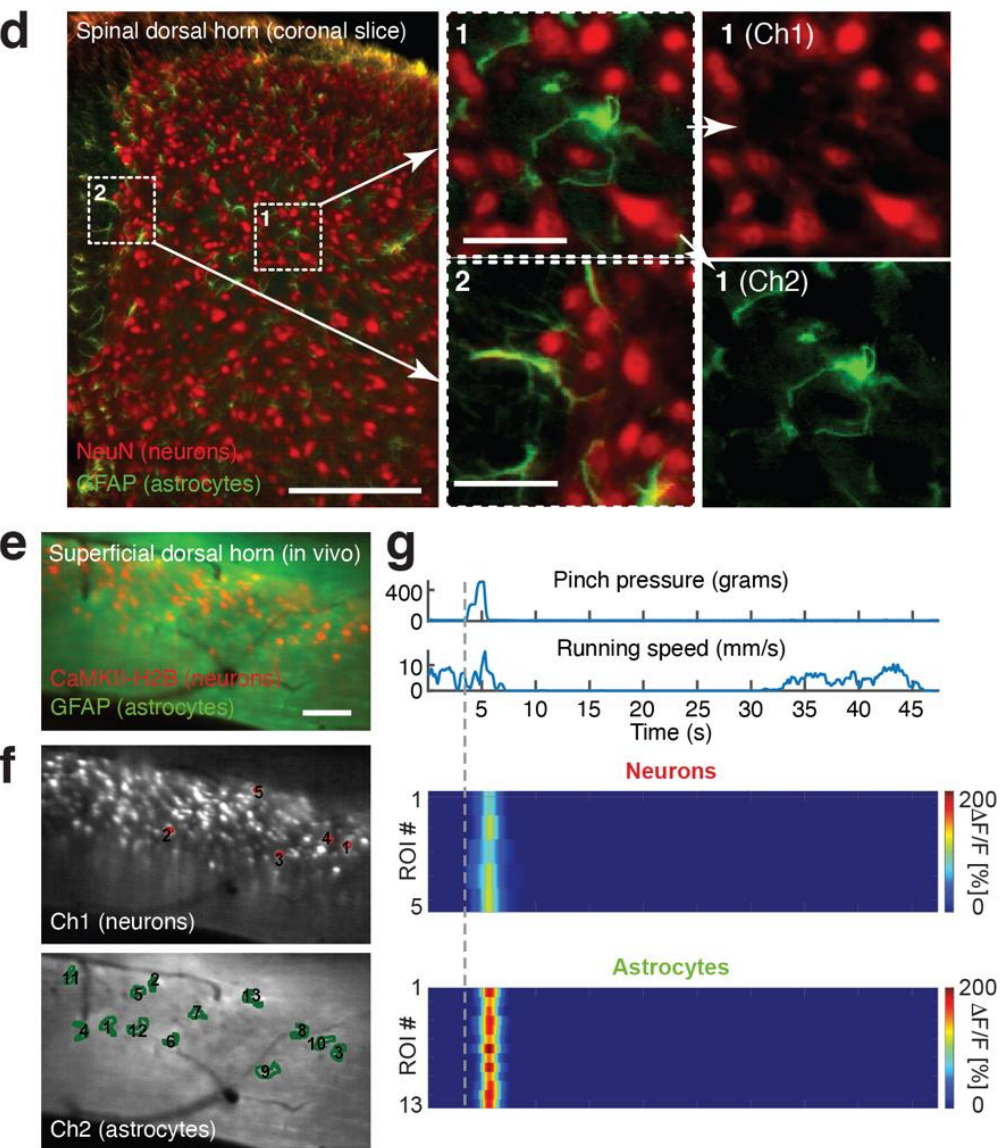

Fig. 2 | Wearable microscopes with custom-compound microlenses permit multi-color

404 polystyrene beads labeled with three distinct fluorophores demonstrating three-color imaging

405 with a single RGB image sensor and dual-band filters. Scale bar, $250 \mu \mathrm{m}$. b-c, Individual color

406 channels before (b) and after (c) color unmixing (Methods). Scale bars, $100 \mu \mathrm{m}$. d, Left,

407 example fluorescence image of a $20-\mu \mathrm{m}$-thick mouse spinal cord section stained for astrocytes

408 (GFAP; green) and neurons (NeuN; red) with two distinct fluorophores (Alexa Fluor 488 and 
409 633) demonstrating two-color imaging in vitro. Scale bar, $250 \mu \mathrm{m}$. Center, blow-up of the two

410 indicated subregions on the left. Scale bars, $50 \mu \mathrm{m}$. Right, color-separated images for subregion

411 1. e, Example fluorescence image from a time-lapse recording showing neuronal nuclei (red)

412 and astrocytes (green) in the spinal dorsal horn of a behaving mouse demonstrating multiplex

413 imaging in vivo. Imaging was performed about three weeks after AAV9-CaMKII-H2B-GCaMP7f-

414 TagRFP injection into the lumbar spinal cord of GFAP-GCaMP6f mice. Scale bar, $100 \mu \mathrm{m} . \mathbf{f}$,

415 Color-separated images with neuronal (red) and astrocyte (green) regions of interest (ROIs)

416 indicated. g, Noxious tail pinch-evoked neuronal nuclear and astrocyte calcium transients in the

417 ROls shown in f. Pressure stimuli were applied to the mouse's proximal tail (Methods). 


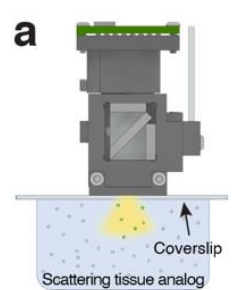

b
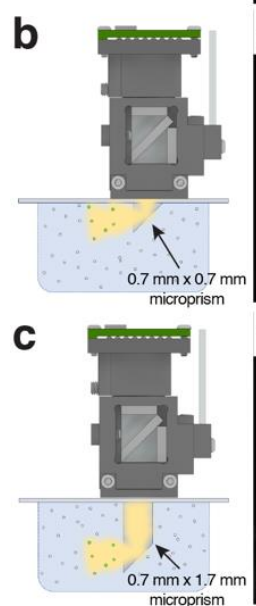
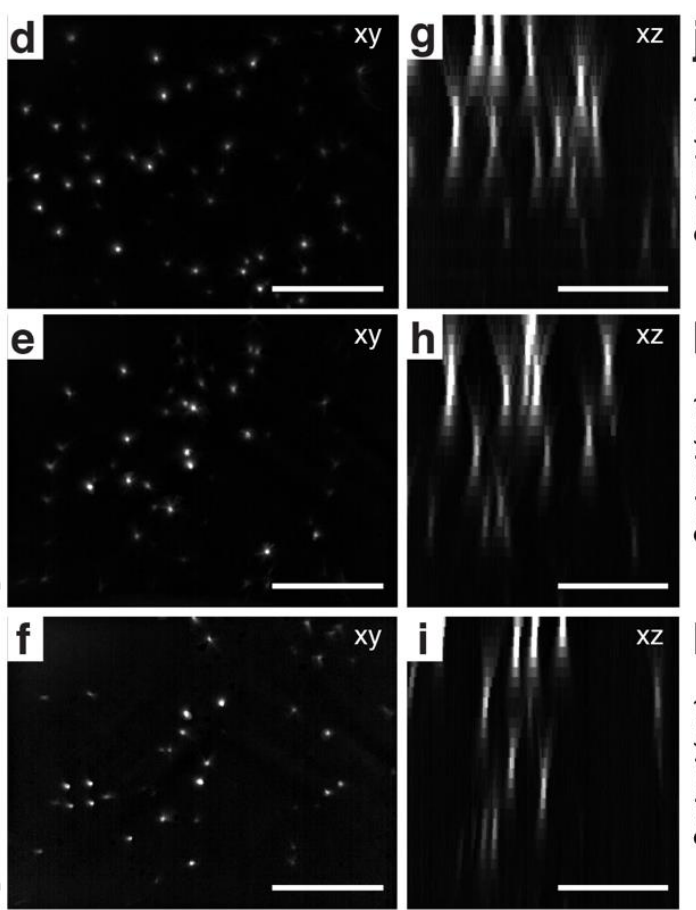
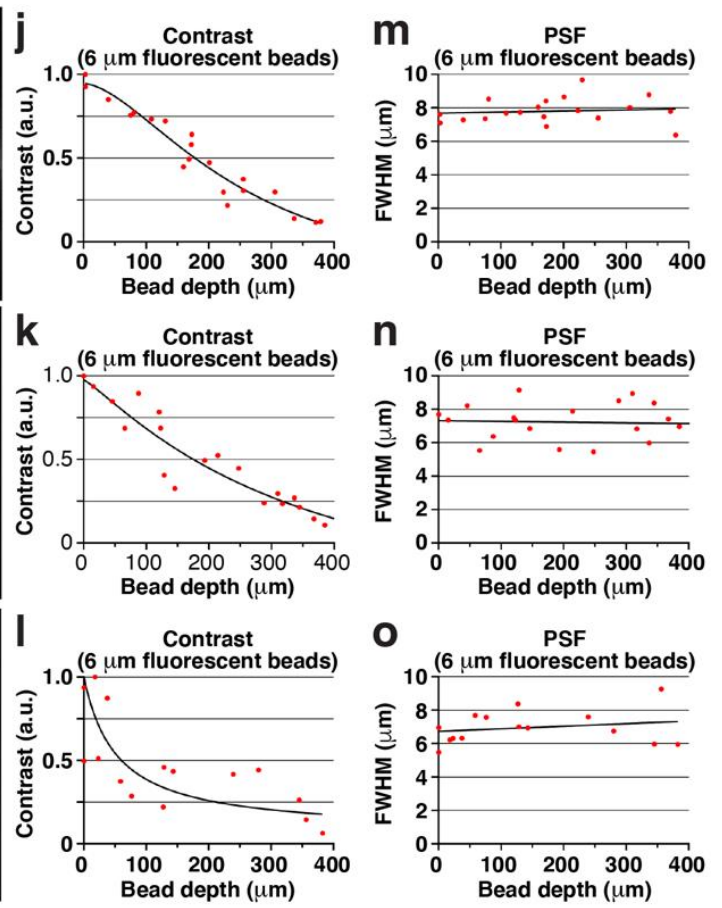

Fig. 3 | Wearable microscopes with custom-compound microlenses permit deep imaging

421 through implanted microprisms. a-c, Schematics showing the experimental approach for

422 characterizing and comparing three imaging conditions in scattering tissue phantoms

423 (Methods): Top, imaging through a coverslip; center, imaging through a coverslip with an

424 attached $0.7 \mathrm{~mm} \times 0.7 \mathrm{~mm} \times 0.7 \mathrm{~mm}$ microprism $(\mathrm{W} \times \mathrm{D} \times \mathrm{H})$; bottom, imaging through a

425 coverslip with an attached $0.7 \mathrm{~mm} \times 0.7 \mathrm{~mm} \times 1.7 \mathrm{~mm}$ microprism. d-f, example images of

426 tissue phantom embedded $6 \mu \mathrm{m}$-diameter fluorescent beads. Each image is a maximum

427 intensity projection through a z-stack acquired as shown in a-c by translating the microscope

428 axially. Scale bar, $250 \mu \mathrm{m}$. g-i, maximum intensity side projections of the acquired z-stacks.

429 Scale bar, $150 \mu \mathrm{m}$. j-I, bead contrast as a function of imaging depth. $\mathbf{m}-\mathbf{0}$, lateral FWHM of the 6 $430 \mu$ m-diameter fluorescent beads as a function of imaging depth. 


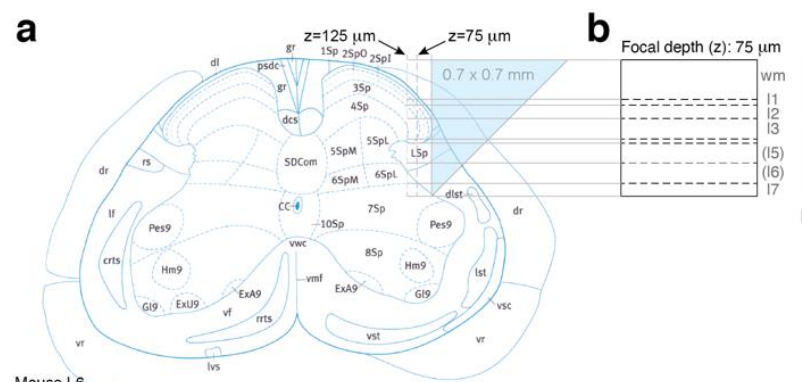

d

Noxious pinch trial without run

$\square=50 \% \Delta \mathrm{F} / \mathrm{F} \quad \square=75 \% \Delta \mathrm{F} / \mathrm{F}$

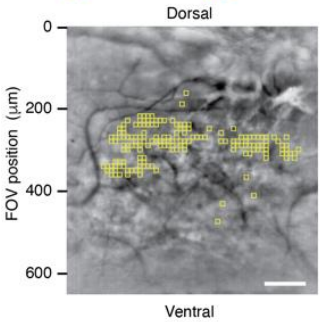

i

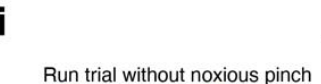

$\square=50 \% \Delta \mathrm{F} / \mathrm{F} \quad \square=75 \% \Delta \mathrm{F} / \mathrm{F}$

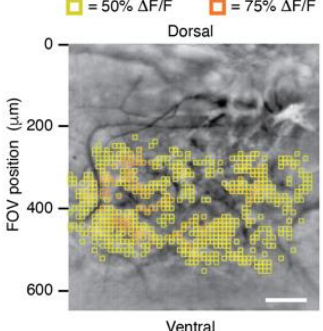

e
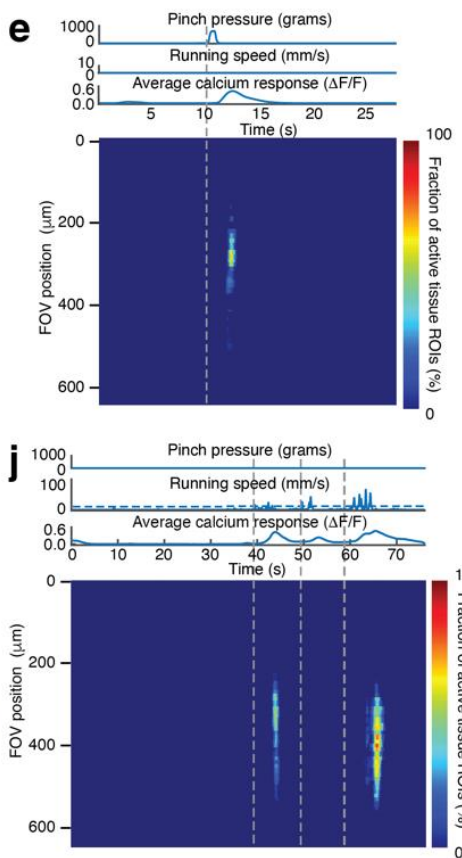

C

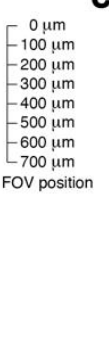

$\mathbf{f}$
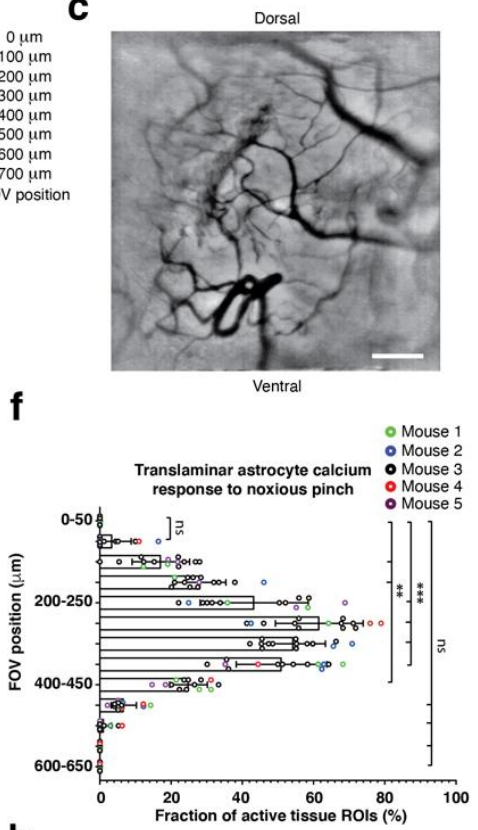

k

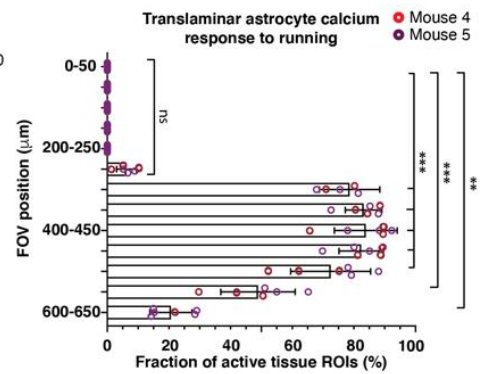

g

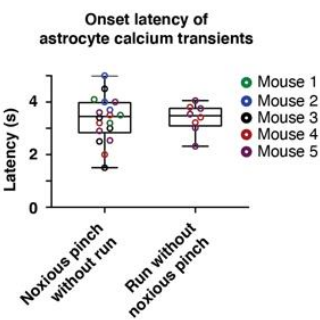

h

Duration of astrocyte calcium transients (noxious pinch without run)

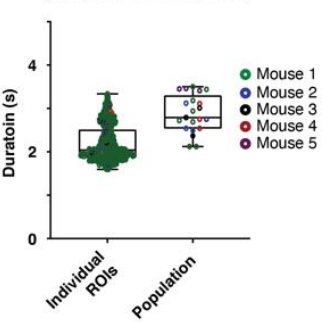

I

Duration of astrocyte calcium transients
(run without pinch)

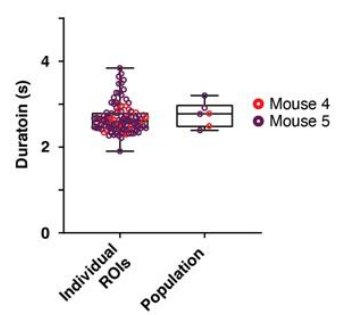

433 Fig. 4 | High-speed translaminar imaging reveals region-specific sensory and motor

imaging approach. An $0.7 \mathrm{~mm} \times 0.7 \mathrm{~mm} \times 0.7 \mathrm{~mm}$ glass reflective microprism was implanted at

the lateral interface between the spinal gray and white matter at the L4-L5 spinal level

437 (Methods). The microprism's reflective hypotenuse tilts the microscope's imaging plane by 90

438 degrees allowing high-speed measurements across laminae. Imaging was performed at 75 or

$439125 \mu \mathrm{m}$ focal depth from the vertical microprism face. Scale bar, $0.5 \mathrm{~mm}$. b. Predicted extent

440 and borders of spinal laminae across the $700 \mu \mathrm{m}$ field of view (FOV) for a $75 \mu \mathrm{m}$ focal depth. c,

441 Example fluorescence image showing the translaminar blood vessel pattern in a GFAP-

442 GCaMP6f mouse at $75 \mu \mathrm{m}$ focal depth four weeks after microprism implantation. Scale bar, 100 
$443 \mu \mathrm{m} . \mathbf{d}$, Average intensity projection image from a different GFAP-GCaMP6f mouse at $75 \mu \mathrm{m}$

444 focal depth overlaid with $10 \mu \mathrm{m} \times 10 \mu \mathrm{m}$ ROIs. Only ROls with at least $50 \%$ (yellow) or $75 \%$

$445 \Delta F / F$ (orange) in response to a noxious tail pinch ( $p>500 \mathrm{~g}$; duration: $1.5 \mathrm{~s} \pm 0.5 \mathrm{~s})$ are shown.

446 In this example, the tail pinch did not evoke a locomotor response. Scale bar, $100 \mu \mathrm{m}$. e,

447 Noxious tail-pinch evoked activity across tissue depth and time for the example recording shown

448 in $d$. Each row depicts the percent of active ROIs ( $\geq 50 \% \Delta F / F)$ for a given tissue depth. The

449 corresponding pressure stimulus, locomotor activity, and average calcium transient across the

450 FOV are shown above the activity heat map. Running speed was recorded by placing the

451 animal on a spherical treadmill. $\mathbf{f}$, Population data showing the average percent of active ROls

452 across tissue depths. Significant activity occurred in central FOV regions, corresponding to the

453 upper spinal laminae. g, Population data of average calcium response onset latency for pinch-

454 only and run-only trials. h, Population data showing individual ROI and average calcium

455 transient duration for pinch-only trials. i, Activity map from the same animal as in $d$ for a

456 spontaneous run trial without pressure stimulus application. Same focal depth as in d. Scale

457 bar, $100 \mu \mathrm{m}$. j, Running-evoked astrocyte activity across tissue depth and time. The pressure

458 sensor readout, locomotor activity, and average calcium signal across the FOV are shown

459 above the activity heat map. k, Population data showing the average percent of active ROls

460 across tissue depths for run trials. Significant activity occurred in FOV regions corresponding to

461 deep spinal laminae involved in motor processing. I, Population data showing individual ROI

462 and average calcium transient duration for run-only trials (2-4 s-long runs). The data in $\mathrm{f}, \mathrm{g}$ (left),

$463 \mathrm{~h}$ is from 1,105 ROls, 18 recordings, and 5 mice. The data in $\mathrm{g}$ (right), $\mathrm{k}, \mathrm{I}$ are from $855 \mathrm{ROIs,} 8$

464 recordings, and 2 mice. All shown data were acquired four weeks after microprism implantation.

465 Paired t-tests determined $P$ values, and all bar plots are presented as mean \pm s.e.m. The box

466 and violin plots mark the median and the 25th and 75th percentiles, and the whiskers cover the

467 minimum and maximum of the data. 
a

Noxious pinch trial without run

$\square=25 \% \Delta \mathrm{F} / \mathrm{F} \quad \square=50 \% \Delta \mathrm{F} / \mathrm{F}$ Dorsal

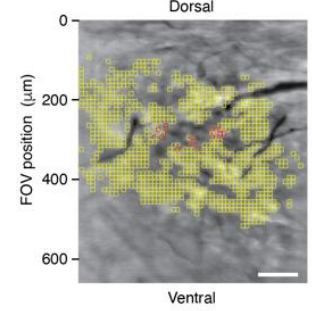

f

Run trial without noxious pinch

$\square=25 \% \Delta \mathrm{F} / \mathrm{F} \quad \square=50 \% \Delta \mathrm{F} / \mathrm{F}$

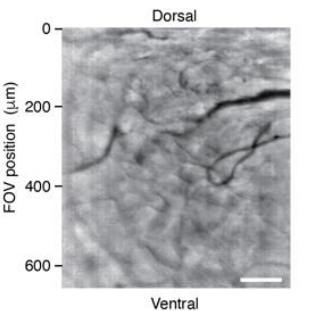

Ventral
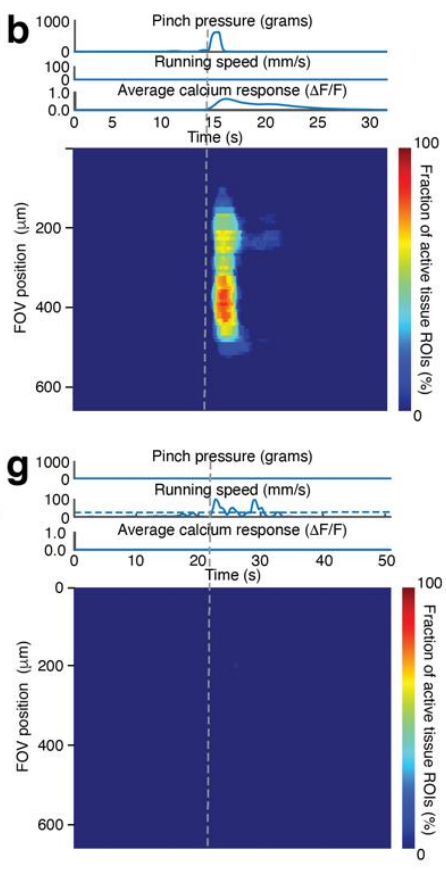

C

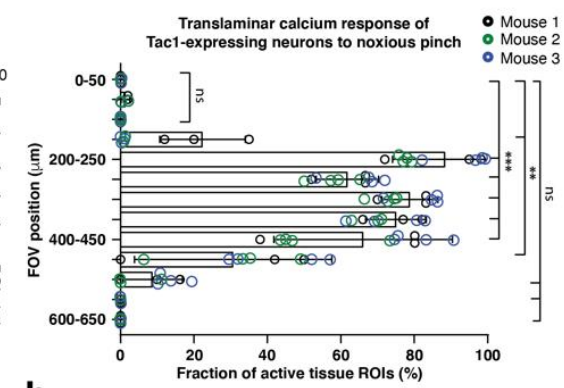

h

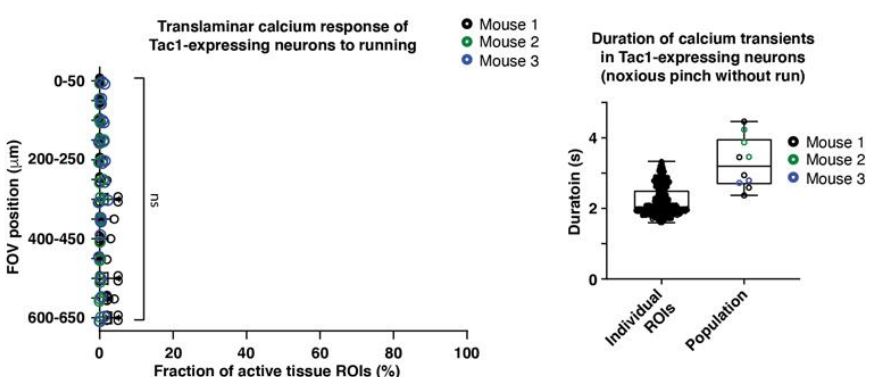

Fig. 5 | High-speed translaminar imaging reveals noxious mechanical stimulus-evoked

activity in the spinal cord of behaving Tac1-GCaMP6f mice. a, Average intensity projection

471 image from a Tac1-GCaMP6f mouse overlaid with $10 \mu \mathrm{m} \times 10 \mu \mathrm{m}$ ROls. Only ROls with at least

$47225 \%$ (yellow) or $50 \% \Delta \mathrm{F} / \mathrm{F}$ (orange) in response to a noxious tail pinch ( $\mathrm{p}>500 \mathrm{~g}$; duration: 1.5

$473 \mathrm{~s} \pm 0.5 \mathrm{~s})$ are shown. In this example, the tail pinch did not evoke a locomotor response. The

474 focal depth was $75 \mu \mathrm{m}$. Scale bar, $100 \mu \mathrm{m}$. b, Noxious tail-pinch evoked activity across tissue

475 depth and time for the example recording shown in a. Each row depicts the percent of active

476 ROls ( $\geq 25 \% \Delta \mathrm{F} / \mathrm{F})$ for a given tissue depth (Methods). The applied pressure stimulus,

477 locomotor activity, and average calcium transient across the FOV are shown above the activity

478 heat map. c, Population data showing the average percent of active ROls across tissue depths.

479 Significant activity occurred in FOV regions corresponding to the upper spinal laminae. d,

480 Population data showing the average calcium transient onset latency for pinch-only trials. e,

481 Population data showing individual and average calcium transient duration for pinch-only trials.

482 f, Activity map from the same animal as in a for a spontaneous run trial without pressure

483 stimulus application. Same focal depth as in a. Scale bar, $100 \mu \mathrm{m} . \mathbf{g}$, Running-evoked activity 
484 across tissue depth and time. The pressure sensor readout, locomotor activity, and average

485 calcium signal across the FOV are shown above the activity heat map. $\mathbf{h}$, Population data

486 showing the average percent of active ROls across tissue depth for run-only trials. No

487 significant calcium activity occurred within the FOV in response to running. The data in c, $d, e$

488 are from 1,299 ROls, 11 recordings, and 3 mice, while the data in $\mathrm{h}$ are from 8 recordings in 3

489 mice. All shown data were acquired four weeks after microprism implantation. Paired t-tests

490 determined $P$ values, and all bar plots are presented as mean \pm s.e.m. The box and violin plots

491 mark the median and the 25th and 75th percentiles, and the whiskers cover the minimum and

492 maximum of the data. 


\section{Methods}

496 Miniature microscope design. Our wearable microscopes include six major components: the

497 custom optics barrels (including six microlenses), fluorescence filter cube, main body, image

498 sensor module, illumination module, and base plate (Fig. 1a).

Optical design. The optical design was performed using Zemax optical modeling software and manual calculations. During the design phase, geometric distortion was deliberately allowed to increase to $5.7 \%$ to gain improvement across the other Seidel aberrations (spherical, coma, astigmatism, and field curvature). Our final optical design utilized six lens elements, each from a

504 different type of glass and housed in two separate barrels (Optics Technology Inc.).

The filter cube consisted of a custom $4.2 \times 6.84$ x $1 \mathrm{~mm}$ dual-band dichroic beamsplitter (59012custom, Chroma Technology Corp.), $4.1 \times 4.2 \times 1.1 / 1.0 \mathrm{~mm}$ dual-band fluorescence excitation and emission filters (59012x and 59012m, Chroma Technology Corp.), and two uncoated $4.2 \mathrm{x}$ $4.2 \times 4.2 \mathrm{~mm}$ fused silica prisms (Tower Optical Corp.). This all-glass filter cube configuration was designed to correct the lateral beam path offset and optical aberrations introduced by the

511 dichroic beamsplitter. Dichroic beamsplitters are typically designed for 45-degree incidence from

512 air. We, therefore, designed a custom coating that matched the $59012 \mathrm{bs}$ transmission spectrum

513 allowing the filter to be used for a 45-degree incidence from glass. Filter cube assembly was

514 done manually using index-matched UV-cure adhesive (NOA146H, Norland Products Inc.) to

515 minimize internal reflections. The outside of the filter cube was painted with a custom black

516 paint (black pigment dispersed in $\mathrm{NOA} 146 \mathrm{H}$, Norland Products Inc.) to absorb stray light

517 reflections. A retainer ring was introduced to reduce filter cube-related optical aberrations (e.g.,

518 color shifts) at the edges of the FOV. This ring decreased the effective NA from 0.46 to 0.41 , a $519 \sim 21 \%$ reduction in the light collection. 
521 The illumination module was designed to hold a hand-polished poly(methyl methacrylate)-core

522 (PMMA-core) multi-mode fiber (MMF) with $735 \mu \mathrm{m}$ core and $0.51 \mathrm{NA}$ (Eska, Mitsubishi

523 International PolymerTrade Corp.). We selected a PMMA- instead of a silica-core MMF because

524 its flexibility aided the animal's mobility. The large core size and NA provided efficient light

525 coupling (e.g., from LEDs). The illumination module also included a $1.4 \mathrm{~mm} 45^{\circ}$-fold-mirror,

526 constructed from two $45^{\circ}-45^{\circ}-90^{\circ} 1 \times 1 \times 1 \mathrm{~mm}$ micro-prisms with a reflective-coated

527 hypotenuse (Tower Optical Corp.), mounted inside the module. The fold mirror was coupled to

528 the optical fiber using index-matched UV-cure adhesive (NOA81, Norland Products Inc.), while

529 the collector lens (45-664, Edmund Optics) was secured inside the microscope's main body.

530 The fold mirror allowed the MMF to be vertically coupled to the horizontal collector lens, an

531 intentional design to also aid the animal's mobility.

As the illumination sources, we either used two high-intensity table-top LEDs or lasers. The LED

534 light source consisted of blue (470nm) and yellow (554nm) mounted LED packages (M470L4

535 and MINTL5, Thorlabs) combined in free-space and coupled into a silica-core intermediary MMF

536 patch-cord (M59L01, ThorLabs). The patch-cord was then mated to the illumination module's

537 MMF using an SMA to SMA mating sleeve (ADASMA, ThorLabs). The purpose of the

538 intermediary MMF patch-cord was to allow for the precise alignment of the LEDs to be

539 unaffected when switching light sources (e.g., between the LEDs and lasers). A $240 \mu \mathrm{m}$ core

540 MMF (Eska, Mitsubishi International PolymerTrade Corp.) was used with table-top lasers (473

$541 \mathrm{~nm}$ and $556 \mathrm{~nm}$ DPSS lasers; MBL-FN-473 and MGL-FN-556, respectively; CNI

542 Optoelectronics Tech. Co.).

544 Optomechanical design. The housing included four modules (main body, sensor mount,

545 illumination module, and base plate) and was designed in CAD software (Autodesk Inventor).

546 This modular design simplifies the optimization process and allows for future upgrades without 
547 the need to develop an entirely new device. The main body holds the optics barrels, the filter

548 cube, and the illumination collector lens. It was designed with an open central compartment to

549 access the interchangeable fluorescence filter cube easily. The main body also includes

550 features for mating with the sensor mount, base plate, and illumination modules. The sensor

551 mount holds the image sensor and blocks stray light. It contains threading and a locking

552 setscrew to allow fine focus adjustments after mounting. The illumination module was designed

553 to provide flexibility in using LED or laser light sources in multiplex imaging applications. The

554 detachable base plate allows reproducible mounting, facilitating chronic imaging experiments.

555 For ease of fabrication, three-axis computer numerical control (CNC) milling was selected. This

556 technique influenced the design process as the complex geometry needed to be confined to

557 dimensions accessible to the CNC mill. Most housing components were fabricated from the

558 lightweight polyether ether ketone (PEEK) (Zera Development Company). The sensor mount

559 was fabricated from aluminum to aid in dissipating the heat generated by the image sensor.

561 Electronic design. We selected a CMOS sensor (MT9M024, Aptina Imaging) that matches our

562 optical resolution (3.75 um pixel spacing, corresponding to $0.69 \mu \mathrm{m}$ at the object plane; $1280 \mathrm{x}$

563960 pixels) and offers high sensitivity (5.48 and 6.7 V/lux-sec for the RGB and monochrome

564 version, respectively) and frame rate (45 fps at full resolution). The sensor was mounted on a

565 custom miniature printed circuit board (PCB), interfaced with an Aptina data-acquisition board

566 (AGB1N0CS-GEVK), and controlled using the Aptina DevWare Software Package. For high-

567 speed multi-color imaging (at $45 \mathrm{fps}$ ), we used an RGB version of the sensor. For high-

568 sensitivity imaging through microprisms, we used its monochrome version. All data were

569 recorded with 12-bit depth.

571 Microscope characterization. Optical system performance was characterized before any 
572 software-based post-processing (e.g., spatial down-sampling, PCA, or ICA). Image resolution

573 was quantified via the point spread function (PSF) and the limit of resolution (LOR) test (Fig. 1d-

574 e; Fig. 3d-i, m-o; Suppl. Fig. 1). To measure the imaging optics' PSF, we designed a test rig

575 that secured the optics barrels in place. It also contained a simulant filter block (i.e., a glass

576 blank of the same thickness and composition as the fluorescence filter block) and spacer on the

577 object side to ensure a working distance of $\sim 2 \mathrm{~mm}$ to the target. A Zeiss LSM 780 confocal

578 microscope was used to image sub-resolution $(0.5 \mu \mathrm{m})$ fluorescent beads through the optics

579 barrels. To measure the integrated system's PSF, we generated a point source of light using a

$580 \quad 0.5 \mu \mathrm{m}$ pinhole (TC-RT01, Technologie Manufaktur), green fluorescence reference slide (2273,

581 Ted Pella), and blue LED excitation light source (M470L3, Thorlabs). We then took images in

582 evenly spaced axial intervals around the focus point of the assembled microscope. PSF values

583 were calculated from lateral and axial maximum intensity image stack projections and are

584 specified as the full width at half maximum (FWHM) (Fig. 3d-i, m-o; Suppl. Fig. 1).

To measure the LOR at the center and different locations across the FOV $(210 \pm 20 \mu \mathrm{m}$

587 spacing), we recorded images of a high-resolution microscopy USAF target (TC-RT01,

588 Technologie Manufaktur) with a maximum spatial frequency of $3300 \mathrm{lp} / \mathrm{mm}$ (Fig. 1d-e). The

589 focus was set at the center and kept stable across the radial positions to account for field

590 curvature and astigmatism. We plotted the line profile across the bar variance at each recording

591 location and averaged along the bar length. We then averaged the peaks and troughs and

592 calculated the contrast percentage using the Michelson contrast equation. This data analysis

593 utilized the "Find Peaks" ImageJ plugin. We defined the LOR limit (highest spatial frequency) by

594 the bar set where at least $5 \%$ contrast can be observed.

595

596 The contrast was quantified via the modulation transfer function (MTF) (Suppl. Fig. 2). Contrast

597 is the object's brightness relative to the background calculated via the Michelson contrast 
equation. To measure the MTF, we employed the Slanted Edge test using a razor blade, green

599 fluorescence reference slide (2273, Ted Pella), and blue LED excitation light source (M470L3,

600 Thorlabs). The focus was set at the center of the FOV and kept stable across different radial

601 positions (the same positions as in the LOR test). Corresponding data were analyzed using the

602 "Slanted Edge MTF" ImageJ plugin. We calculated both the sagittal and tangential MTF. While

603 MTF $0 \%$ is frequently reported, MTF values below $10 \%$ are affected by sensor noise limits and

604 optical phase reversal. MTF spatial frequency is therefore reported at $10 \%$, mimicking biological

605 samples of a specific labeling density. Corresponding line widths are equal to one-half the

606 spatial period.

608 The FOV was measured by imaging a grid target, printed directly on the surface of the 609 microscope slide without glass coverslip, and using a precision actuator (DRV3, Thorlabs). To

610 demonstrate the multi-color imaging capability of our integrated device, we imaged cell body-

611 sized $(15 \mu \mathrm{m})$ fluorescent polystyrene microspheres of different colors (yellow-green F8844,

612 yellow F21011, and red F8842 beads with 505 nm/515 nm, 515 nm/534 nm, 580 nm/605 nm

613 excitation/emission peaks; ThermoFisher) (Fig. 2a-c). Corresponding data were processed in

614 DevWare using the demosaicing algorithm "Anisotropic Diffusion."

616 The working distance (i.e., the distance from the edge of the lower optics barrel to the object

617 plane) was measured by placing the microscope in contact with a grid target (R1L3S3P,

618 Thorlabs) and then translating it upwards with a precision differential actuator (DRV3, Thorlabs)

619 until the image was in focus. The working distance depends on the position of the image sensor

620 along its focusing range and the thickness of the cover glass. We used a \#0 glass coverslip

621 ( 100 $\mu \mathrm{m}$ thickness) for our measurements and positioned the image sensor in the middle of its 622 focusing track. 
624 The weight of the integrated system was determined by weighing the assembled device,

625 including the custom optics barrels, filters, illumination module, housing, image sensor, and

626 sensor PCB. We did not include the sensor wires or optical fiber in the measurement because

627 external mounts or a commutator typically support the weight of these tether components.

629 Experimental model and subject details. All procedures were performed following the

630 National Institutes of Health $(\mathrm{NIH})$ guidelines and were approved by the Institutional Animal

631 Care and Use Committee (IACUC) at the Salk Institute. Mouse strains used in this study

632 included GFAP-Cre (RRID: IMSR_JAX:012886), Tac1-Cre (RRID: IMSR_JAX:021877), and

633 Ai95(RCL-GCaMP6f)-D mice (RRID: IMSR_JAX:024105) ${ }^{29-31}$. Mice were group-housed,

634 provided with bedding and nesting material, and maintained on a 12-h light-dark cycle in a

635 temperature $\left(22 \pm 1^{\circ} \mathrm{C}\right)$ and humidity controlled (45-65\%) environment. All the imaging and

636 behavioral experiments involved 6-11 weeks-old heterozygous male and female mice.

637 Experimental mice used in individual experiments typically originated from different litters. Mice

638 had marks for unique identification. No criteria were applied to allocate mice to experimental 639 groups.

641 Molecular cloning, AAV production, and titering. The cDNA for H2B-GCaMP7f-TagRFP was

642 PCR amplified and subcloned into an AAV transfer vector downstream of the CaMKIla promoter

643 and upstream of WPRE and hGHpA sequences. This vector was co-transfected into HEK293-

644 AAV cells (Vector Biolabs) along with a pAdeno-helper vector and a pRC-AAV9 rep-cap

645 plasmid. Recombinant AAV9 production was then carried out using a protocol developed by the 646 Byungkook Lim laboratory at UCSD. The recombinant AAV9-CaMKIla-H2B-GCaMP7f-TagRFP647 WPRE-hGHpA was titered by qPCR using primers designed to the hGHpA sequence. The titer 648 of the virus was $4.9 \mathrm{E}+12 \mathrm{GC} / \mathrm{ml}$. 
650 Stereotactic injections. For dual-color in vivo imaging (Fig. 2e-g), the AAV9-CaMKII-H2B-

651 GCaMP7f-TagRFP vector was injected into the L3-L5 spinal cord (coordinates: ML 0.3, DV

$652 \quad 0.15-0.3 \mathrm{~mm}$; volume: $1 \mu \mathrm{l}$; dilution: 1:25). Surgical procedures closely followed previously

653 established protocols ${ }^{4}$. Briefly, thin-wall glass pipettes were pulled on a Sutter Flaming/Brown

654 micropipette puller (model P-97). Pipette tips were cut at an acute angle under 10x

655 magnification using sterile techniques. Tip diameters were typically $15-20 \mu \mathrm{m}$. Pipettes that did

656 not result in sharp bevels or had larger tip diameters were discarded. Millimeter tick marks were

657 made on each pulled needle to measure the virus volume injected into the spinal cord. Mice

658 were anesthetized with isoflurane (4-5\% for induction; $1 \%-1.5 \%$ for maintenance) and

659 positioned in a computer-assisted stereotactic system with digital coordinate readout and atlas

660 targeting (Leica Angle Two). Body temperature was maintained at $36-37^{\circ} \mathrm{C}$ with a DC

661 temperature controller, and ophthalmic ointment was used to prevent eyes from drying. A small

662 amount of depilator cream (Nair) was used to remove hair at and around the incision site. The

663 skin was then cleaned and sterilized with a two-stage scrub of betadine and $70 \%$ ethanol,

664 repeated three times. Surgical scissors were used to make a small (around $10 \mathrm{~mm}$ ) incision

665 along the dorsal midline. Fascia connecting the skin to the underlying muscle was removed with

666 forceps. The skin was held back by retractors. Using blunt dissection, lateral edges of the spinal

667 column were isolated from connective tissue and muscle. Tissue from the vertebra of interest

668 and one vertebra rostral and caudal to the site of spinal cord exposure was removed with

669 forceps. The spine was then stabilized using Cunningham vertebral clamps, and any remaining

670 connective tissue on top of the exposed vertebrae was removed with a spatula. An

671 approximately $0.3 \mathrm{~mm}$ opening was made in the tissue overlying the designated injection site

672 (e.g., using sterile Dumont \#3 forceps). For injection, a drop of the virus was carefully pipetted

673 onto parafilm (2-3 $\mu \mathrm{l})$ for filling the pulled injection needle with the desired volume. Once loaded 
674 with sufficient volume, the injection needle was slowly lowered into the spinal cord until the

675 target depth was reached. Manual pressure was applied using a 30-ml syringe connected by

676 shrink tubing to slowly inject the viral solution over 5-10 $\mathrm{min}$. The syringe's pressure valve was

677 then locked. The position was maintained for approximately $10 \mathrm{~min}$ to allow the virus to spread

678 and to avoid backflow upon needle retraction. Following the injection, spinal cord clamps were

679 removed, muscle approximated, and the skin sutured along the incision. Mice were given

680 subcutaneous Buprenex SR $(0.5 \mathrm{mg} / \mathrm{kg})$ and allowed to recover before placement in their home 681 cage.

683 Live animal preparation. Animals were implanted with a spinal and head plate under general 684 anesthesia approximately one week before laminectomy, as previously described ${ }^{4}$. Buprenex 685 SR $(0.5 \mathrm{mg} / \mathrm{kg})$ was given to minimize post-operative pain.

687 Multi-color imaging of superficial laminae. A laminectomy (2 wide x $4 \mathrm{~mm}$ long) was performed

688 at the T12-T13 vertebra level, corresponding to spinal segments L3-L5 ${ }^{26}$, four days before the 689 initial recording session. The dura mater overlying the spinal cord was kept intact, and a 690 custom-cut \#0 coverslip was used to seal the laminectomy creating an optical window for 691 imaging. The coverslip was replaced immediately before recording sessions to maximize optical 692 clarity.

693

694 Translaminar imaging through implanted glass reflective microprisms. At least one day before 695 surgery, a $0.7 \times 0.7 \times 0.7 \mathrm{~mm}$ glass microprism with aluminum-coated hypotenuse (cat. no. 696 4531-0021; Tower Optical) was UV-cured (NOA 81; cat. no. 8106; Norland Products Inc.) to a 697 custom-cut \#0 coverslip matching the intended laminectomy size ( $3 \mathrm{~mm}$ wide $\times 4 \mathrm{~mm}$ long). To 698 minimize bubbles at the microprism-coverslip interface, the microprism was moved in a circular 
motion and then positioned at the desired location before UV curing. Excess glue around the

700 microprism was removed with sterile absorbent paper points (cat. no. 50-930-669; Fisher

701 Scientific). After UV curing, the microprism-coverslip assembly was placed in a sterile glass petri

702 dish with a cover and heated on a hot plate at $50^{\circ} \mathrm{C}$ to allow further curing for 8-12 h. Following

703 heat curing, the microprism-coverslip assembly was stored in a 70\% EtOH solution. To implant

704 this assembly, mice were anesthetized with isoflurane (4-5\% for induction and $1-2 \%$ for

705 maintenance in $100 \%$ oxygen) and placed in a stereotactic setup. Body temperature was

706 maintained at $36-37^{\circ} \mathrm{C}$ with a DC temperature controller. A laminectomy $(3 \mathrm{~mm}$ wide $\times 4 \mathrm{~mm}$

707 long) was performed at the T13-L1 vertebra level, corresponding to spinal segments L4-L6 ${ }^{26}$.

708 Using a dissecting knife (cat. no. 10055-12; Fine Science Tools) attached to a stereotactic arm,

709 a small incision was made $0.7 \mathrm{~mm}$ lateral to the central vein's center, coinciding roughly with the

710 interface between the dorsal root ganglia (DRG) and spinal white matter in 6-8 weeks-old mice.

711 The incision extended $0.7 \mathrm{~mm}$ in the rostrocaudal direction and $0.7 \mathrm{~mm}$ in depth, matching the

712 microprism dimensions. Cerebrospinal fluid but not blood briefly exuded from the incision site

713 upon retraction of the dissecting knife. The surgical site was washed several times with cold,

714 sterile saline. The microprism implant, held by an $18 \mathrm{G} \times 1 \frac{1}{2}$ " blunt angled syringe needle (cat.

715 no. 305180; Thermo Fisher Scientific) attached to a vacuum suction line, was positioned above

716 the incision site. The implant was slowly lowered until fully inserted ( 0.7 $\mathrm{mm}$ depth). Excess

717 fluid was removed using sterile absorbent paper points (cat. no. 50-930-669; Fisher Scientific).

718 Once affixed to the surrounding bone with instant adhesive (cat. no. 3EHP2; Grainger), the

719 suction holding the implant was turned off.

\section{$721 \quad$ Fluorescence imaging}

722 In vivo imaging through a dorsal optical window. Data in behaving mice were acquired following

723 previously established protocols ${ }^{4}$. Fiber-coupled blue $(470 \mathrm{~nm})$ and yellow $(554 \mathrm{~nm})$ LEDs 
724 (M470L4 and MINTL5, Thorlabs) were used for GCaMP7f and TagRFP excitation (Fig. 2e-g).

725 Approximately 10-20 recordings were taken per imaging session, with each recording lasting

726 around 1-2 min. The typical average power for a given recording and light source was $<125 \mu \mathrm{W}$

$727 \mathrm{~mm}^{-2}$. No signs of phototoxicity, such as a gradual increase in baseline fluorescence, lasting

728 changes in activity rate, or blebbing of labeled cells, were apparent in our recordings.

730 In vivo imaging through implanted microprisms. Data in behaving mice were acquired up to four

731 weeks after microprism implantation (Figs. 4-5). The miniature microscopes' FOV allowed

732 imaging of the entire microprism face. Recordings were taken at $75 \mu \mathrm{m}$ and $125 \mu \mathrm{m}$ focal depth

733 from the vertical microprism-tissue interface, where $0 \mu \mathrm{m}$ was defined as the point when tissue

734 blood vessels first came into focus. A fiber-coupled $473 \mathrm{~nm}$ DPSS laser was used for GCaMP6f

735 excitation. Each imaging session included approximately 10-20 recordings, with each recording

736 lasting around 1-2 min. The typical average light power at the tissue surface was between 275-

$737325 \mu \mathrm{W} \mathrm{mm}{ }^{-2}$. All in vivo data was acquired at the image sensor's full resolution $(1,280 \times 960$

738 pixels) and maximum frame rate $(\sim 45 \mathrm{~Hz})$.

740 Confocal imaging of stained tissue sections. Three-channel, 3 × 3 tiled z-stacks (15 images at 1

$741 \mu \mathrm{m}$ axial spacing) were acquired with a Zeiss LSM 710 confocal microscope to produce images

742 of whole spinal cord sections (laser lines: 405 nm, 488 nm, 633 nm). Each image within the z-

743 stacks had a 1,024 x 1,024-pixel resolution and was acquired using an Olympus 20x 0.8 NA air-

744 matched objective.

746 Sensory stimuli and behavioral tests

747 Sensory stimuli during in vivo imaging. Each imaging session consisted of up to 20 recordings.

748 In a subset of recordings, mechanical stimuli were delivered to the animal's tail using a rodent 
749 pincher system (cat. no. 2450; IITC Life Science, Inc.). Pinch pressures were applied in the

750 dorsoventral direction at approximately $6 \mathrm{~mm}$ from the base of the animal's tail. Each pinch

751 stimulus (typically one per recording) lasted around 1-2 s. Subsequent stimuli were delivered at

752 least 1.5-2 minutes apart to minimize response adaptation. The order in which stimuli of

753 different amplitudes were delivered was randomized. Figs. 2e-g, 4-5 show data from $p>500$

754 mechanical stimuli.

755

756 Sensory tests before and after microprism implantation. To quantify the effects of microprism

757 implantation on the animal's sensory performance, we used the von Frey assay (Suppl. Fig. 6a-

758 b). The Simplified Up-Down (SUDO) method $^{32}$ was performed using Touch Test Sensory

759 Evaluators (North Coast), starting on the sixth $(0.4 \mathrm{~g})$ fiber. The SUDO Method utilizes five

760 stimulations per test, ensuring each animal undergoes the same number of hind paw

761 stimulations. Animals were habituated to the behavioral testing room for at least 15 minutes in

762 their home cage, then to the von Frey apparatus for at least 45 minutes with the researcher

763 outside of the room, and the researcher's presence for an additional 15 minutes. Stimulations

764 were performed every 5 minutes, alternating between hind paws. Four female GFAP- and Tac1-

765 GCaMP6f mice were tested before and 7 days after spinal plate implantation and again at two

766 and four weeks after microprism implantation. Data from the two-week time point are an

767 average of trials performed on days 13 and 15, whereas data from the four-week time point are

768 an average of trials conducted on days 27 and 29 after microprism implantation. Animals were

769 tested twice to decrease the effect of inter-day noise, with a rest day between trials. In vivo

770 microscopy was performed on days 14 and 28. The researcher was blinded to the microprism

771 implantation side (left or right side of the spinal cord).

772

773 Motor tests before and after microprism implantation. To quantify the effects of microprism 
774 implantation on the animal's locomotor performance, we used the kinematic weight-bearing test

775 (Bioseb) (Suppl. Fig. 6c-f). Animals were acclimated to the testing environment by placing their

776 home cage in the testing room approximately 30 minutes before the recordings. The animals

777 were then transferred to the behavior setup, allowing them to explore the new environment for

778 about 15 minutes freely. Following this period, five runs were collected per animal and for five

779 animals total. Runs were counted only if the animal moved from left to right in the setup without

780 stopping. Stride length $(\mathrm{cm})$, peak force $(\mathrm{cN})$, and running speed $(\mathrm{cm} / \mathrm{s})$ were analyzed. Runs of

781 a given animal were averaged. The same animals were measured before and after spinal plate

782 implantation, and two and four weeks after microprism implantation.

784 Immunohistochemistry. Two or four weeks after microprism implantation, mice were

785 euthanized in their home cage following American Veterinary Medical Association (AVMA)

786 guidelines. Transcardial perfusion was performed using $10 \%$ sucrose followed by $4 \%$

787 paraformaldehyde. L1-L6 spinal cord tissue was carefully extracted and incubated in 4\% PFA

788 overnight at $4^{\circ} \mathrm{C}$. The tissue was then washed on a shaker with $1 \times$ PBS three times with 15 min

789 per cycle, followed by incubation in $30 \%$ sucrose in PBS overnight at $4^{\circ} \mathrm{C}$. Next, the tissue was

790 flash-frozen in an OCT tissue-freezing medium, cryosectioned at $20 \mu \mathrm{m}$ thickness, air-dried

791 overnight, and then processed for immunostaining. Tissue sections were incubated overnight at

$79244^{\circ} \mathrm{C}$ with primary antibody diluted in blocking buffer, then washed in PBS containing $0.1 \%$

793 Tween-20, and incubated for two hours at $22-24^{\circ} \mathrm{C}$ in the dark with fluorophore-coupled

794 secondary antibodies. Primary antibodies included NeuN (1:100; Novus Biologicals Inc.; cat. no.

795 NBP1-92693; RRID: AB_11036146), GFAP (1:250; Thermo Fisher Scientific; cat. no. 13-0300;

796 RRID: AB_2532994), and Iba-1 (1:200; FUJIFILM Wako Shibayagi; cat. no. 019-19741; RRID:

797 AB_839504). Secondary antibodies included Alexa Fluor 633 goat anti-mouse (1:100; Thermo

798 Fisher Scientific; cat. no. A-21052; RRID: AB_2535719), Alexa Fluor 488 goat anti-rat (1:100; 
799 Thermo Fisher Scientific; cat. no. A-11006; RRID: AB_2534074), and Alexa Fluor 405 goat-anti-

800 rabbit (1:100; Thermo Fisher Scientific; cat. no. A-31556; RRID: AB_221605).

801

802 Image data processing and analysis

803 In vivo imaging data. We used a custom Matlab script to convert the image sensor's raw files

804 from 12- to 16-bit, demosaic corresponding images (for RGB image sensor data only), and

805 adjust their pixel range. The data were then further processed in Fiji. We converted the image

806 data to TIFF, cropped the time-lapse recordings to the LED-/laser-on period and fluorescently

807 labeled central areas of the FOV, followed by illumination correction, background subtraction,

808 and color separation (for RGB image sensor data only). Full-frame image motion was reduced

809 using $\mathrm{Moco}^{33}$. For multi-color image data, one channel was selected to determine and correct x-

810 y image displacements across all channels. Image edge artifacts introduced by motion

811 correction were cropped. Within-frame distortions were typically small and therefore not

812 corrected. Motion-corrected calcium imaging data were analyzed using custom ImageJ and

813 MATLAB software. Manually drawn cell body-sized ROls were used to extract activity traces

814 from neurons and astrocytes in dual-color recordings (Fig. 2e-g). We applied several

815 quantitative exclusion criteria to ensure that the activity traces derived from these ROls are from

816 a defined cell type. Activity traces were considered from neurons if the average TagRFP

817 fluorescence within respective ROls exceeded the average TagRFP fluorescence across the

818 FOV by at least 2 s.d. This retained only AAV9-CaMKII-H2B-GCaMP7f-TagRFP transduced

819 cells with sharp boundaries (i.e., in-focus neurons). Activity traces were considered from

820 astrocytes if the average TagRFP fluorescence within the respective ROls was below 0.5 s.d.

822 To quantify calcium activity more comprehensively across the FOV, we used an unbiased tiling

823 approach, similar to our previous work ${ }^{34}$ (Figs. 4-5). Each tile corresponded to a $10 \times 10 \mu \mathrm{m}$

824 ROI. We defined three ROI classes: over, near, and distant from blood vessels. First, we 
825 calculated the mean and s.d. of the recording's average intensity image. Next, we determined

826 individual ROIs' average intensity values (A). If $A<$ mean - s.d., it was considered a blood

827 vessel-ROI. If $A \geq$ mean $+(1+x)^{*}$ s.d. (with $x \in[0.3-0.7]$ depending on data set (e.g., blood

828 vessel size/pattern)), it was classified as distant from blood vessels. ROls with in-between

829 values were considered near-blood vessel-ROls. ROls over and near blood vessels were

830 excluded from data analysis. For all ROls distant from blood vessels, we calculated their

831 average fluorescence intensity over time. Corresponding activity traces were temporally

832 smoothed using an $0.4 \mathrm{~s}$ sliding average. Local maxima within the traces were identified using

833 Matlab's "findpeaks" function. The fluorescence baseline and noise level (in s.d.) of a given

834 trace were calculated using a $2 \mathrm{~s}$ period before stimulus or run onset. Local maxima were

835 considered evoked activity if fluorescence intensity values surrounding the peak were at least 6

836 s.d. above baseline for $\geq 2 \mathrm{~s}$. Calcium transient onset was defined as the point at which the

837 fluorescence intensity trace immediately before the peak crossed a 2 s.d. threshold above

838 baseline. Calcium transient duration was defined as the trace's full width at half maximum

839 (FWHM). Traces that showed a) activity within $2 \mathrm{~s}$ after recording onset (i.e., before stimulus or

840 run onset), b) fluorescence decreases faster than the indicator's unbinding kinetics ( $\geq 50 \%$ signal

841 drop within $\leq 250 \mathrm{~ms}$ ), or c) a 3 s.d. drop below fluorescence baseline after transient onset for $\geq 3$

842 s were considered artifactual (e.g., caused by tissue motion) and excluded from further analysis.

843 For multi-peak transients, the largest peak's value was used for FWHM calculation. If the

844 intensity values between peaks fell below the half-maximum value for $\geq 250 \mathrm{~ms}$, the half-

845 maximum point closest to the largest peak was used for FWHM calculation. If the trace's

846 amplitude fell below 2 s.d. above baseline for $\geq 400 \mathrm{~ms}$, the corresponding transients were

847 considered separate calcium spikes.

848

849 To distinguish spontaneous from evoked calcium activity, we applied additional criteria. A

850 calcium transient was considered evoked if its onset occurred within $1 \mathrm{~s}$ or $5 \mathrm{~s}$ after stimulus- or 
run-onset for neurons or astrocytes with cytosolic GCaMP expression, respectively. For nuclear

852 GCaMP expression, we allowed a $5 \mathrm{~s}$ time window. For all activity traces that passed the filters

853 mentioned above, we calculated $\Delta \mathrm{F}(\mathrm{t}) / \mathrm{F}_{0}(\mathrm{t}) . \mathrm{F}_{0}$ was determined with Matlab's mode function

854 using a 10-bin width. Traces that showed abrupt intensity changes $(\geq 0.5 \Delta \mathrm{F} / \mathrm{F}$ within $\leq 65 \mathrm{~ms})$

855 were removed from the analysis. To compare neuronal and astrocyte activity, we plotted

856 corresponding $\Delta \mathrm{F} / \mathrm{F}$ traces aligned on a given cell type with the other cell type being sorted

857 based on its transient onset time.

859 Confocal imaging data. All data were processed, analyzed, and plotted using Fiji, IMARIS, and

860 Prism software. To quantify immuno-stained tissue, we first generated digital representations of

861 the labeled cells/structures using Imaris' (version 9.2; Bitplane) creation wizard. Cells/structures

862 were classified as "Spots" (to quantify the number of NeuN- and lba-1-positive cell bodies;

863 Suppl. Figs. 3, 5) or "Surfaces" (to quantify GFAP volume including cell bodies and major

864 processes; Suppl. Fig. 4). For surface quantifications, we used detail and threshold levels of

8650.1 and 0.4 , respectively, for all tissue slices and hemispheres. These quantifications were

866 performed for various regions of interest (ROIs) (e.g., $50 \times 50 \mu \mathrm{m}$ or $50 \times 700 \mu \mathrm{m})$ (Suppl. Figs.

867 3-5). Volume fractions are represented in percent (Suppl. Fig. 4b-e).

870 recorded at $1 \mathrm{kHz}$ using DAQExpress 2.0 software (National Instruments). Analog data included

871 the pressure sensor output from the rodent pincher system, treadmill speed, and on-off TTL

872 signal of the miniaturized microscope's light source, depending on the experiment. Pinch

873 application and mouse behavior were also recorded on a video camera ( $\geq 20 \mathrm{~Hz}$; Stingray F-

874 033, Allied Vision Technologies). To synchronize imaging with video data, we placed a near-

875 infrared LED within the video camera's FOV, triggered from the microscope's light source drive 
876 signal (TTL pulse). Imaging and analog data were synchronized by recording the on-off TTL

877 signal of the miniaturized microscope's light source together with all other analog data. To relate

878 the animal's locomotor activity more closely to the imaging data, we focally restrained animals

879 on a spherical treadmill equipped with an optical encoder (E7PD-720-118, US Digital), allowing

880 precise readout of running speed. All analog data was processed using custom MATLAB

881 (Mathworks) routines. The pincher and encoder traces were first cropped to the light source-on

882 period. Pressure traces were then quantified with respect to stimulus amplitude and duration.

883 Encoder traces were smoothed using a sliding average (window size: $0.4 \mathrm{~s}$ ). Locomotion onset

884 or offset was defined as the point at which the smoothed running speed exceeded or fell below

$88510 \mathrm{~mm} / \mathrm{s}$. Encoder traces were analyzed concerning running speed, duration, and frequency. If

886 the running speed fell below the $10 \mathrm{~mm} / \mathrm{s}$ threshold for $\geq 750 \mathrm{~ms}$, the local maxima were

887 considered separate running bouts. The video data were cropped to the LED-on period. Videos

888 were scored manually regarding pinch onset and offset. Calcium transient latency was

889 calculated based on these measurements because video recordings provided higher temporal

890 resolution than the pincher traces. For population analysis, data was computationally sorted

891 using custom MATLAB (Mathworks) routines. Only trials with $1.5 \pm 0.5$ s pinch duration, or $3 \pm 1$

892 s run duration were included in the analysis.

\section{Statistical analysis}

895 All data were analyzed and plotted using MATLAB, Excel, or GraphPad Prism software. Paired 896 t-tests were performed to determine FOV regions with significant calcium activity (Fig. 4f, $\mathbf{k}$ and 897 Fig. 5c, h), on data evaluating neuronal loss (Suppl. Fig. 3d-f), astrocyte activation (Suppl.

898 Fig. 4d-e), microglia activation (Suppl. Fig. 5d-e), mouse motor performance (Suppl. Fig. 6d899 f), hind paw sensitivity changes on the implanted side (comparing the 2-week and 4-week time 900 points to baseline), and the potential effect of spinal plate implantation on sensory performance 
901 (Suppl. Fig. 6b). The latter data were collected for each hind paw before (baseline) and seven

902 days after surgery. Two-way ANOVA was performed on data comparing hind paw sensitivity on

903 the microprism-implanted versus the control hemisphere. All data are represented as mean \pm

904 s.e.m. unless indicated otherwise. Group sample sizes were chosen based on power analysis or

905 previous studies. The following convention was used to indicate $P$ values: 'NS' indicates

$906 P>0.05,{ }^{\prime *}$ indicates $0.01<P \leq 0.05$, '**' indicates $0.001<P \leq 0.01$, , ‘**’ indicates $0.0001<P \leq 0.001$,

907 and '****' indicates $P \leq 0.0001$.

908

909 Reporting summary

910 Further information on research design is available in the Research Reporting Summary linked

911 to this paper.

912

913 Data availability

914 The data that support the findings of this study will be deposited in the Brain Image Library (BIL;

915 https://www.brainimagelibrary.org/index.html). They will also be available from the

916 corresponding author upon reasonable request.

918 Code availability

919 The custom Java- and Matlab-based code used to process and analyze the data will be

920 deposited in GitHub. It will also be available from the corresponding author upon reasonable

921 request.

922

923 


\section{References}

92529 Garcia, A. D., Doan, N. B., Imura, T., Bush, T. G. \& Sofroniew, M. V. GFAP-expressing

926 progenitors are the principal source of constitutive neurogenesis in adult mouse

927 forebrain. Nat Neurosci 7, 1233-1241, doi:10.1038/nn1340 (2004).

92830 Madisen, L. et al. A toolbox of Cre-dependent optogenetic transgenic mice for light-

929 induced activation and silencing. Nat Neurosci 15, 793-802, doi:10.1038/nn.3078 (2012).

$93031 \quad$ Harris, J. A. et al. Anatomical characterization of Cre driver mice for neural circuit

931 mapping and manipulation. Front Neural Circuits 8, 76, doi:10.3389/fncir.2014.00076

932 (2014).

93332 Bonin, R. P., Bories, C. \& De Koninck, Y. A simplified up-down method (SUDO) for

934 measuring mechanical nociception in rodents using von Frey filaments. Mol Pain 10, 26,

935 doi:10.1186/1744-8069-10-26 (2014).

93633 Dubbs, A., Guevara, J. \& Yuste, R. moco: Fast motion correction for calcium imaging.

937 Front Neuroinform 10, 6, doi:10.3389/fninf.2016.00006 (2016).

$93834 \quad$ Patriarchi, T. et al. Ultrafast neuronal imaging of dopamine dynamics with designed

939 genetically encoded sensors. Science 360, doi:10.1126/science.aat4422 (2018). 


\section{Acknowledgments}

942 We thank members of the Nimmerjahn lab for comments on the manuscript, the Salk machine

943 shop for technical support, and J. Chambers for mouse colony management. This work was

944 primarily supported by the National Institutes of Health (NIH) grant R01NS108034 (A.N). It was

945 partially supported by the NIH grants U01NS103522, U19NS112959, and U19NS123719 (A.N.),

946 and equipment funds from C. and L. Greenfield. P.S. was supported by a Rose Hills Foundation

947 graduate fellowship and N.A.N. by funds from an NIH T32/CMG Training Grant, Burt and Ethel

948 Aginsky Research Scholar Award, Kavli-Helinski Endowment Graduate Fellowship, and NIH

949 individual predoctoral fellowship (F31NS120619). The content is solely the authors'

950 responsibility and does not necessarily represent the official views of the NIH.

\section{$951 \quad$ Author contributions}

952 P.S., E.M.C., and A.N. conceived and designed the study. P.S. developed and characterized 953 the wearable microscopes and wrote ImageJ-based data analysis code. E.M.C. conducted the 954 in vivo microprism, motor behavior, and immunostaining experiments. D.D. performed the in 955 vivo multiplex imaging experiments. A. Ngo and G.G. developed Matlab- and ImageJ-based 956 data analysis code, respectively. N.A.N. performed the sensory tests. C.L.K. produced the viral 957 vectors. A.N. supervised the study and wrote the initial manuscript draft. All authors contributed 958 to the text and figures, discussed the results, or provided input and edits on the manuscript.

\section{Competing interests}

960 The authors declare no competing interests.

962 Correspondence and requests for materials should be addressed to A.N. 


\section{Supplementary Figures}

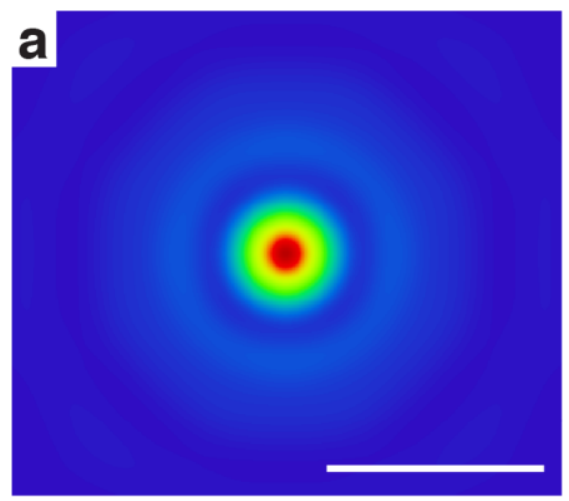

Modeled optical system PSF (X-axis)

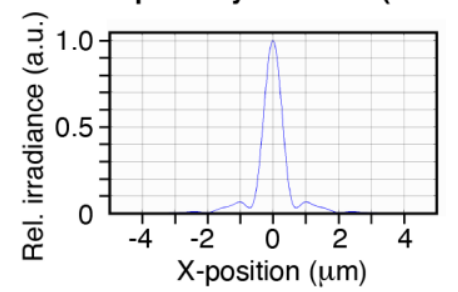

Modeled optical system PSF (Y-axis)

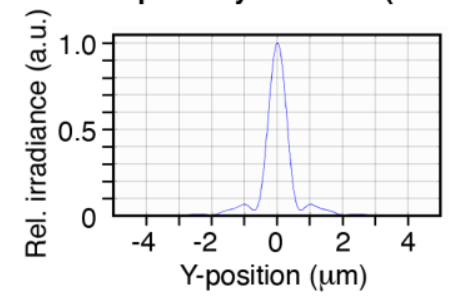

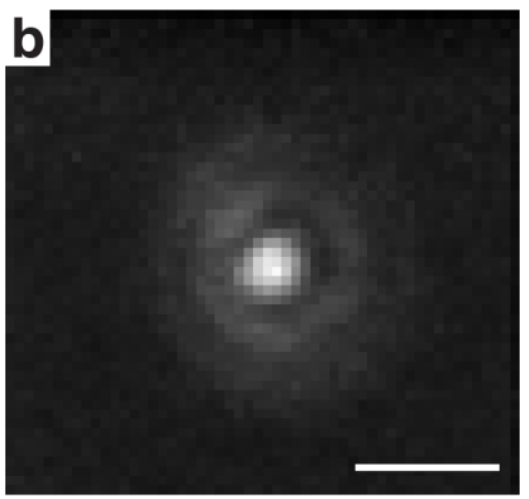

Fab. optical system PSF (X-axis)

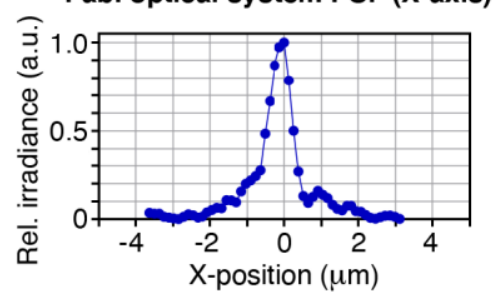

Fab. optical system PSF (Y-axis)

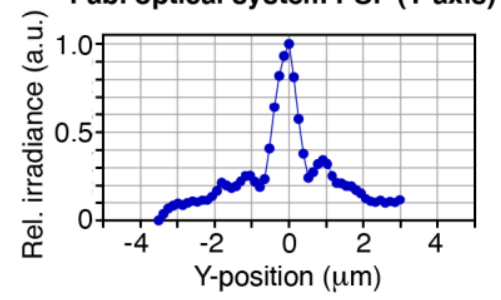

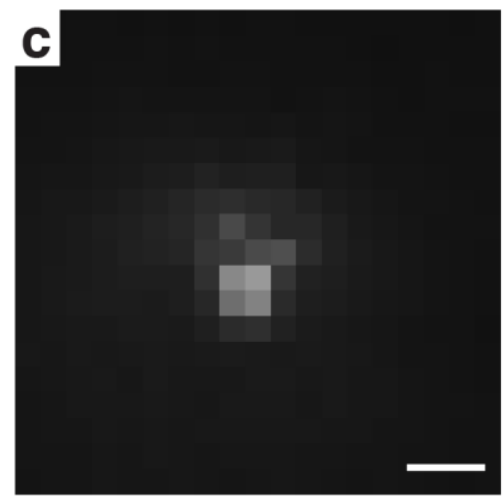

Int. optical system PSF (X-axis)

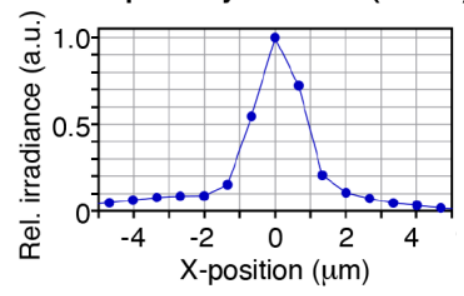

Int. optical system PSF (Y-axis)

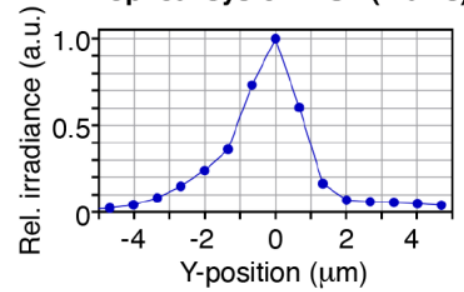

Suppl. Fig. 1 | Wearable microscopes with custom-compound microlenses provide $<1.5$

$\mu \mathrm{m}$ lateral resolution. a, Top, the image shows the optical system's lateral point spread function (PSF) as predicted by Zemax optical modeling. Center, x cross-section. Bottom, y cross-section. b, Top, experimentally measured lateral PSF of the fabricated optical system.

971 Center, $\mathrm{x}$ cross-section. Bottom, y cross-section. c, Top, experimentally measured lateral PSF

972 of the integrated microscope. Center, $\mathrm{x}$ cross-section. Bottom, y cross-section. Scale bars, 2

$973 \mu \mathrm{m}$. 

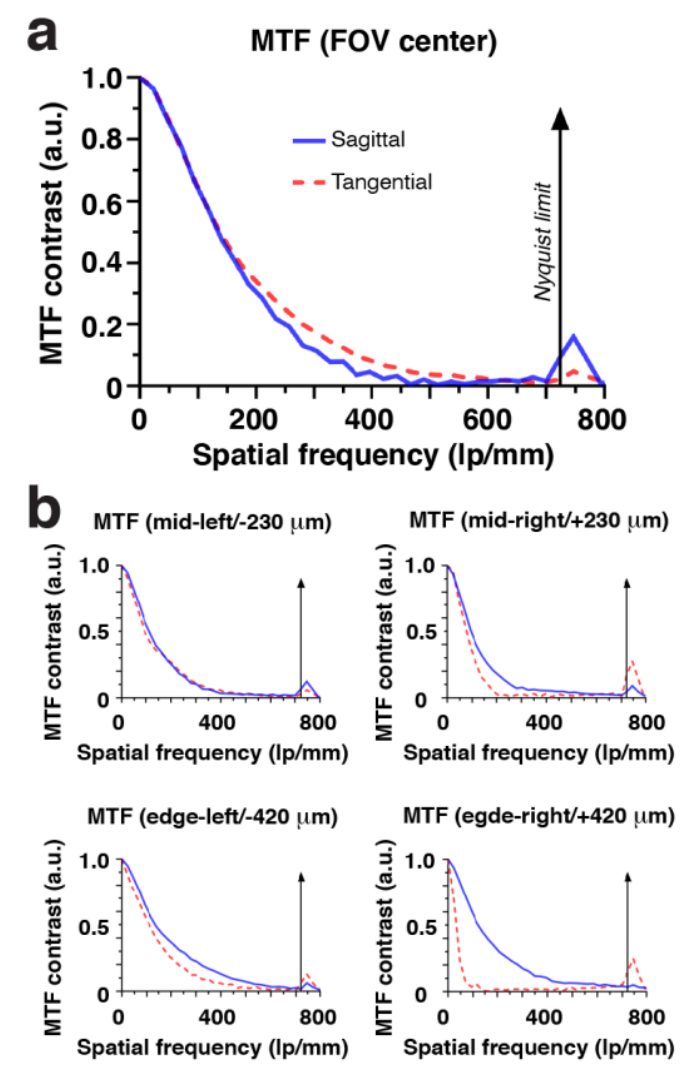

\section{C \\ MTF10 (across FOV)}

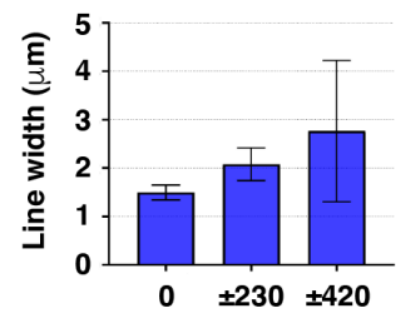

Distance from FOV center $(\mu \mathrm{m})$

977 Suppl. Fig. 2 | Wearable microscopes with custom-compound microlenses provide high

978 contrast across the field of view. a, Modulation transfer function (MTF) of the integrated

979 microscope measured in the center of the field of view (FOV) using the Slanted Edge test. b,

980 MTF at different indicated FOV positions relative to the center. c, MTF contrast at 10\% (MTF10)

981 across the FOV. Displayed values are averages across similar FOV locations and horizontal

982 and vertical Slanted Edge targets. Spatial frequencies were converted to line widths. The data

983 are presented as mean \pm s.e.m. The larger error bars toward the FOV edge likely indicate

984 sample tilt. 


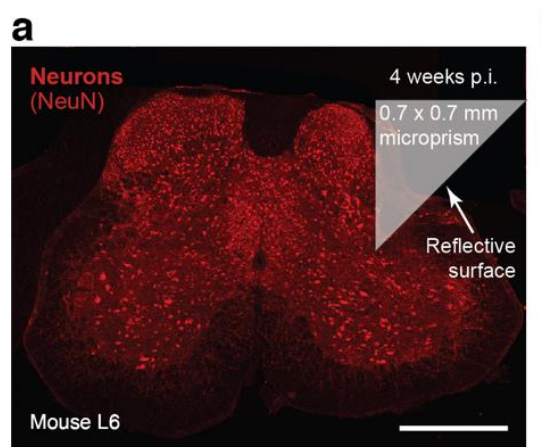

b NeuN-positive cell count -2 weeks p.i. (analysis regions: $50 \times 50 \mu \mathrm{m}$ )

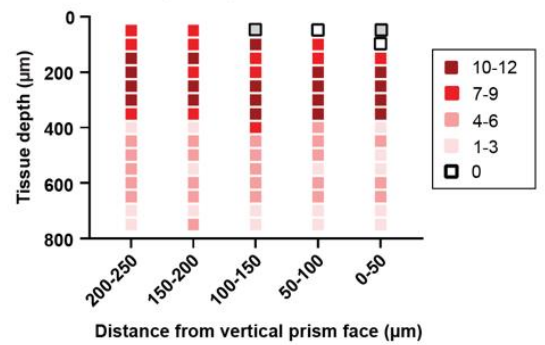

f NeuN-positive cell count

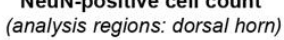

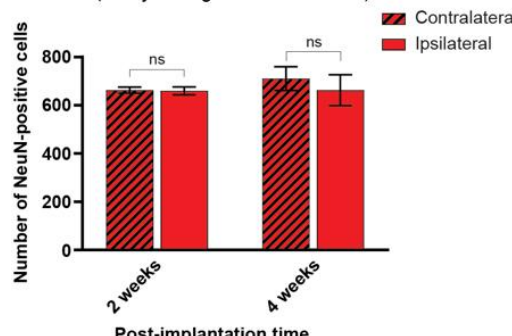

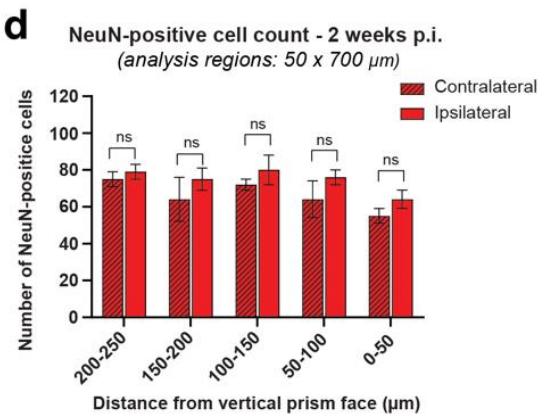

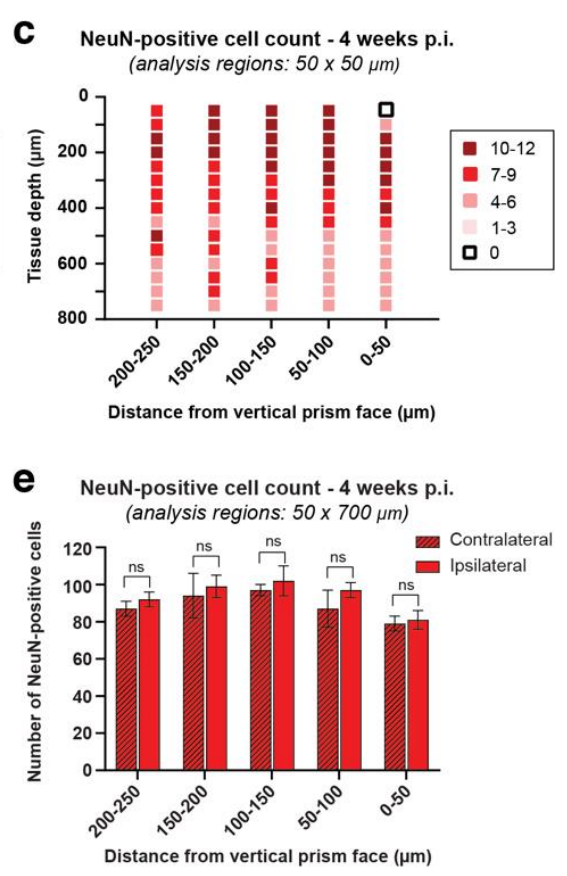

Suppl. Fig. 3 | Microprism implantation does not result in a significant loss of dorsal horn

neurons. a, Fluorescence image showing NeuN-positive cells (neurons) in a 20- $\mu$ m-thick mouse spinal cord section four weeks after microprism implantation. The approximate

991 microprism location is indicated. Scale bar, $500 \mu \mathrm{m}$. b-c, Average NeuN-positive cell count in 50 $992 \mu \mathrm{m} \times 50 \mu \mathrm{m}$ analysis regions plotted as a function of tissue depth and distance from the vertical 993 microprism face two (b) and four weeks after microprism implantation (c). Cell density 994 decreases with depth because of fewer cells with larger cell bodies. Depending on white matter 995 thickness, cell density may also decrease toward the vertical microprism face. d-e, Average 996 NeuN-positive cell count in $50 \mu \mathrm{m} \times 700 \mu \mathrm{m}$ strips plotted as a function of distance from the 997 vertical microprism face two (d) and four weeks after microprism implantation (e). The data are 998 compared to equivalent regions on the non-implanted (contralateral) hemisphere. f, Average

999 NeuN-positive cell count across the entire dorsal horn two and four weeks after microprism 1000 implantation. The data are compared to equivalent regions on the non-implanted (contralateral) 
bioRxiv preprint doi: https://doi.org/10.1101/2021.12.23.474039; this version posted December 24, 2021. The copyright holder for this preprint (which was not certified by peer review) is the author/funder, who has granted bioRxiv a license to display the preprint in perpetuity. It is made available under aCC-BY-NC-ND 4.0 International license.

1001 hemisphere. The data in b-f are from 12 slices and 3 mice for each time point. Paired t-tests

1002 determined $P$ values, and all data are presented as mean \pm s.e.m.

1003

1004 

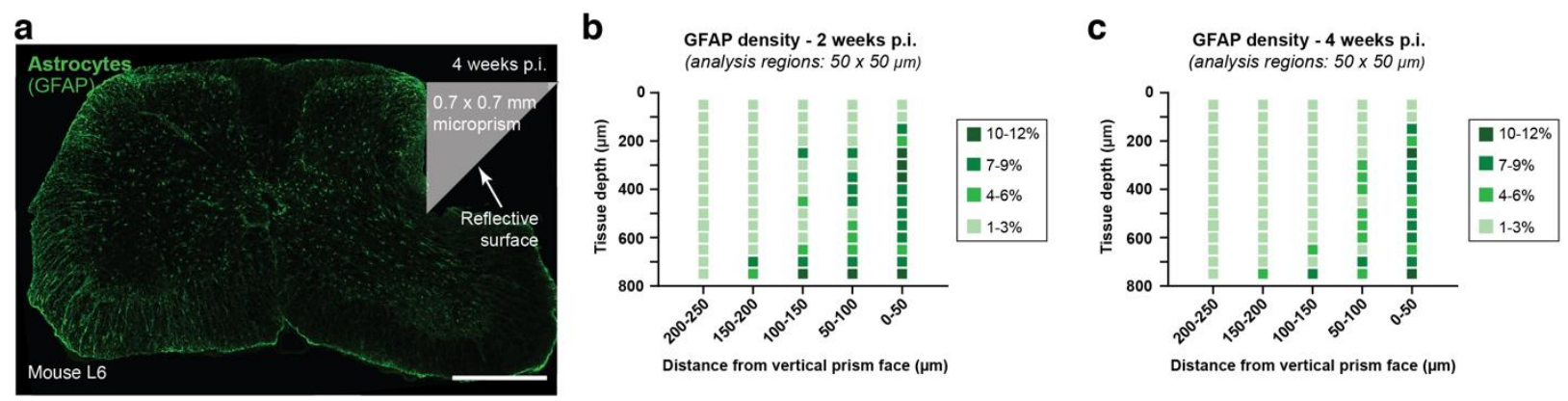

1006
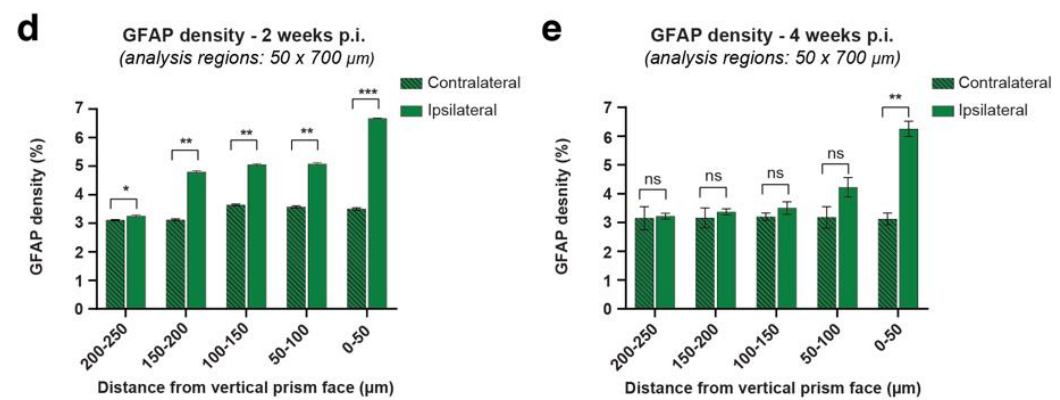

1007 Suppl. Fig. 4 | Microprism implantation leads to a transient glial fibrillary acidic protein

(GFAP) upregulation near the microprism-tissue interface. a, Fluorescence image showing

GFAP staining (astrocytes) in a $20-\mu$ m-thick mouse spinal cord section four weeks after

microprism implantation. The approximate microprism location is indicated. Scale bar, $500 \mu \mathrm{m}$.

1011 b-c, Average GFAP density in $50 \mu \mathrm{m} \times 50 \mu \mathrm{m}$ analysis regions plotted as a function of tissue

1012 depth and distance from the vertical microprism face two (b) and four weeks after microprism

1013 implantation (c). GFAP immunoreactivity decreases over time and is highly localized to regions

1014 immediately adjacent to the vertical microprism-tissue interface. d-e, Average GFAP density in

$101550 \mu \mathrm{m} \times 700 \mu \mathrm{m}$ strips plotted as a function of distance from the vertical microprism face two (d)

1016 and four weeks after microprism implantation (e). The data are compared to equivalent regions

1017 on the non-implanted (contralateral) hemisphere. The data in b-e are from 12 slices and 3 mice

1018 for each time point. Paired t-tests determined $P$ values, and all data are presented as mean \pm

1019 s.e.m. 

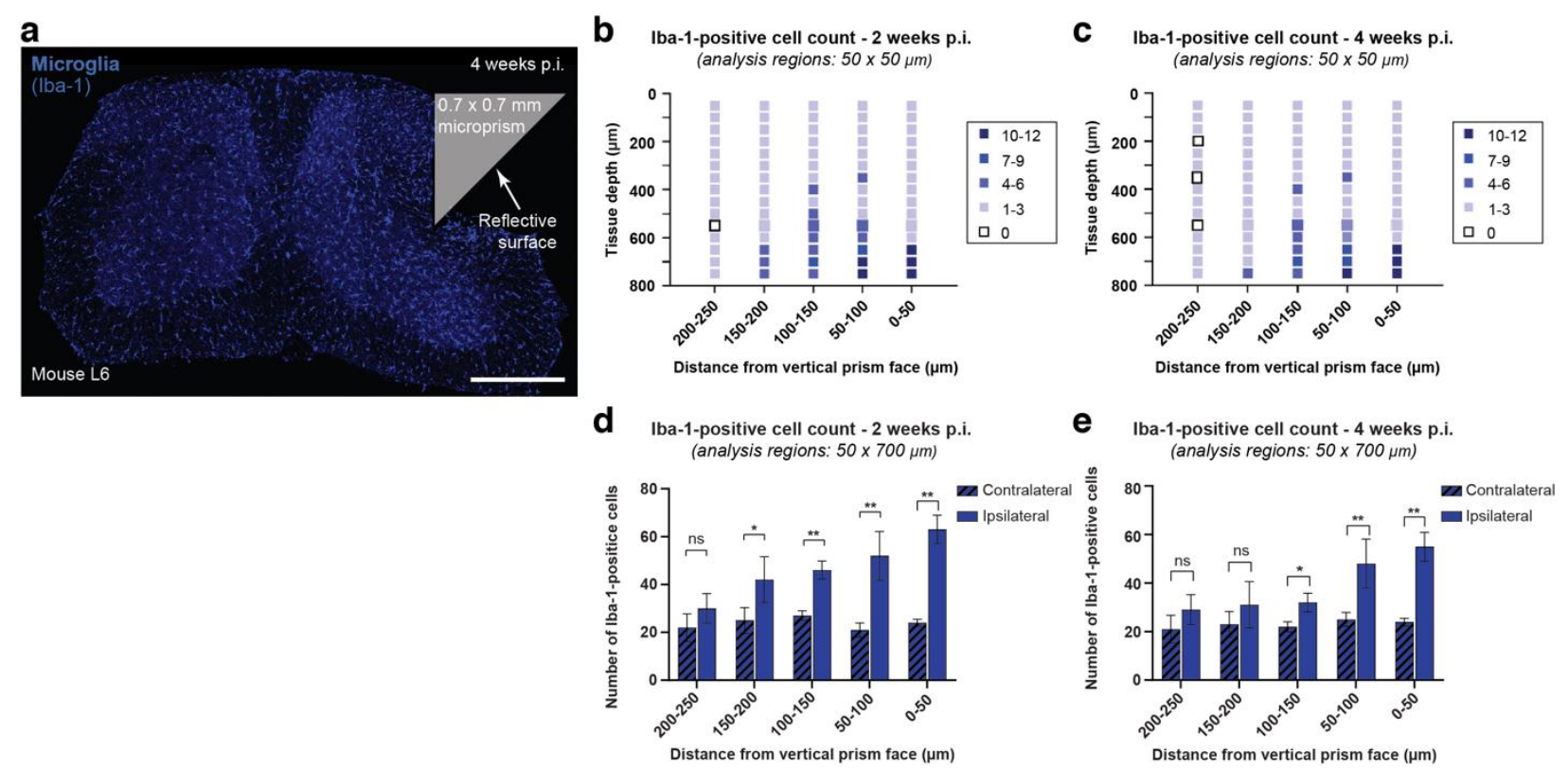

1022 Suppl. Fig. 5 | Microprism implantation leads to a transient and highly localized Iba-1

1023 upregulation near the implanted microprism edge. a, Fluorescence image showing Iba-1-

1024 positive cells (microglia/macrophages) in a $20-\mu m$-thick mouse spinal cord section four weeks

1025 after microprism implantation. The approximate microprism location is indicated. Scale bar, 500

$1026 \mu \mathrm{m}$. b-c, Average lba-1-positive cell count in $50 \mu \mathrm{m} \times 50 \mu \mathrm{m}$ analysis regions plotted as a

1027 function of tissue depth and distance from the vertical microprism face two (b) and four weeks

1028 after microprism implantation (c). Iba-1-positive cell count decreases over time and is highly

1029 localized to regions surrounding the implanted microprism edge. d-e, Average lba-1-positive cell

1030 count in $50 \mu \mathrm{m} \times 700 \mu \mathrm{m}$ strips plotted as a function of distance from the vertical microprism

1031 face two (d) and four weeks after microprism implantation (e). The data are compared to

1032 equivalent regions on the non-implanted (contralateral) hemisphere. The data in b-e are from 12

1033 slices and 3 mice for each time point. Paired t-tests determined $P$ values, and all data are

1034 presented as mean \pm s.e.m. 
a

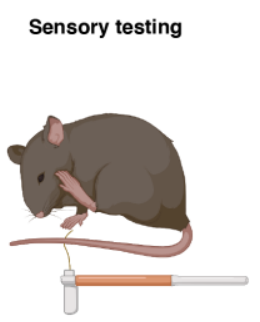

C
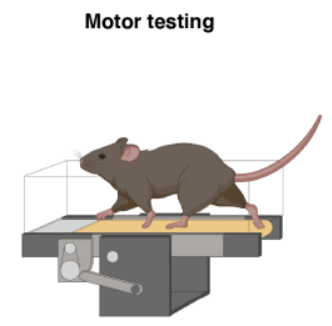

d

d Running speed
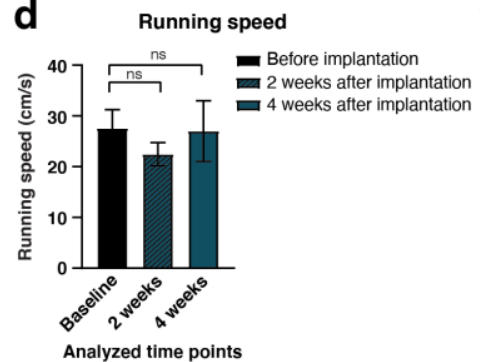

b Von Frey mechanical stimulus sensitivity

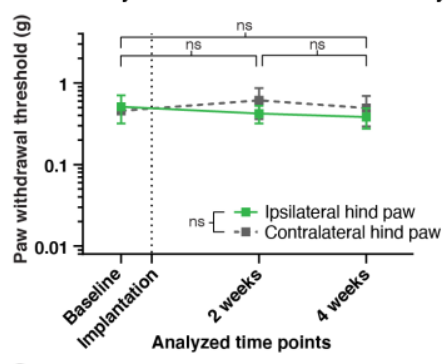

e Stride length

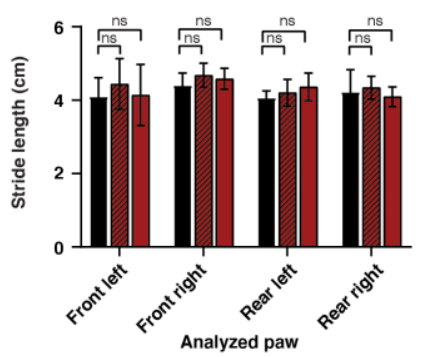

f

Peak force

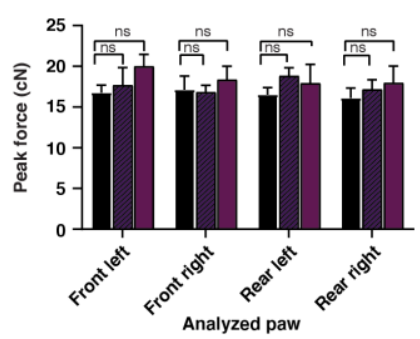

Suppl. Fig. 6 | Microprism implantation does not result in overt sensory or motor deficits.

a, Schematic showing the von Frey assay used for sensory testing. Hind paw withdrawal threshold was quantified for the implanted (ipsilateral) and non-implanted (contralateral) side before and at different time points after microprism implantation at the L4-L5 spinal level. b,

1041 Hind paw withdrawal threshold on the implanted (ipsilateral) and non-implanted (contralateral) side before, two, and four weeks after microprism implantation. Paw withdrawal threshold was not significantly different between time points and hemispheres ( $N=4$ mice; ipsilateral baseline: $0.511 \pm 0.193$; two-week time point: $0.422 \pm 0.103$; four-week time point: $0.383 \pm 0.108) . P$ values were determined by Bonfierri-Dunn corrected paired t-tests between time points for the ipsilateral paw $(P>0.05)$ and two-way ANOVA across all time points between the contralateral and ipsilateral hind paws $(P=0.62)$. The average value of two test sessions is reported for each 
1049 test used for motor testing. d-f, Population data showing running speed (d), front and hind paw

1050 stride length (e), and front and hind paw peak force (f) before, two, and four weeks after

1051 microprism implantation at the L4-L5 spinal level. Locomotor performance was not significantly

1052 different between time points ( $N=4$ mice). All animals were tested at each indicated time point

1053 (Methods). Paired t-tests determined $P$ values, and all data are presented as mean \pm s.e.m. 
1055 Supplementary Tables

\begin{tabular}{llllll}
\hline Surface & Type & Radius & Thickness & Glass & Diameter \\
\hline OBJ & STANDARD & Infinity & 0.0000000 & & 6.0000000 \\
1 & STANDARD & Infinity & 4.5000000 & & 4.8000000 \\
2 & STANDARD & -13.65635 & 1.0000000 & N-SF57 & 2.6790000 \\
3 & STANDARD & 1.758689 & 0.9479822 & & 2.3490000 \\
4 & STANDARD & -10.38426 & 1.0000000 & N-BALF5 & 2.6570000 \\
5 & STANDARD & 9.049044 & 1.1821360 & N-LASF40 & 3.9880000 \\
6 & STANDARD & -3.253426 & 0.6096153 & & 3.7850000 \\
7 & STANDARD & Infinity & 5.0000000 & FSILICA & 4.0000000 \\
8 & STANDARD & Infinity & 0.0000000 & & 4.0000000 \\
9 & STANDARD & Infinity & 0.4398171 & & 2.0000000 \\
STO & STANDARD & 5.543071 & 1.0000000 & N-SF66 & 2.0000000 \\
11 & STANDARD & 1.973004 & 1.1777090 & N-PK51 & 1.9911980 \\
12 & STANDARD & -5.628253 & 0.1000000 & & 2.9940000 \\
13 & STANDARD & 2.635428 & 1.1278330 & N-LAK34 & 3.2970000 \\
14 & STANDARD & -13.04288 & 2.0000000 & & 3.3600000 \\
15 & STANDARD & Infinity & 0.1000000 & D263M & 0.8690751 \\
IMA & STANDARD & Infinity & & & 0.8293949 \\
\hline
\end{tabular}

1056 Suppl. Table 1 | Wearable microscope optical design. 


\section{Supplementary Movies}

Suppl. Movie 1 | High-speed translaminar imaging of tail pinch-evoked calcium activity in behaving GFAP-GCaMP6f mice. Example time-lapse recording acquired with the wearable microscope showing noxious tail pinch-evoked calcium excitation in spinal astrocytes. The data were obtained at $\sim 42.3 \mathrm{fps}$ and $\sim 75 \mu \mathrm{m}$ focal depth from the vertical tissue-microprism interface four weeks after microprism implantation. Elapsed time is indicated in the upper right corner

1064 (total duration: 30 s). To allow a precise readout of locomotor activity, the mouse was placed on a spherical treadmill. Noxious pinch triggered coordinated calcium excitation in the central field of view regions corresponding to upper dorsal horn laminae. Scale bar, $100 \mu \mathrm{m}$.

\section{Suppl. Movie 2 | High-speed translaminar imaging of motor-evoked calcium activity in}

1069 behaving GFAP-GCaMP6f mice. Example time-lapse recording acquired with the wearable

1070 microscope showing running-evoked calcium excitation in spinal astrocytes. The data were 1071 obtained at $\sim 40.3 \mathrm{fps}$ and $\sim 75 \mu \mathrm{m}$ focal depth from the vertical tissue-microprism interface four 1072 weeks after microprism implantation. Elapsed time is indicated in the upper right corner (total 1073 duration: $1 \mathrm{~min} 19 \mathrm{~s})$. To allow a precise readout of locomotor activity, the mouse was placed on 1074 a spherical treadmill. Running triggered coordinated calcium excitation in the lower field of view regions likely corresponding to spinal premotor areas. Scale bar, $100 \mu \mathrm{m}$. behaving Tac1-GCaMP6f mice. Example time-lapse recording acquired with the wearable microscope showing noxious tail pinch-evoked calcium excitation in spinal Tac1-expressing neurons. The data were obtained at $\sim 43.2 \mathrm{fps}$ and $\sim 75 \mu \mathrm{m}$ focal depth from the vertical tissue-

1081 microprism interface four weeks after microprism implantation. Elapsed time is indicated in the 1082 upper right corner (total duration: 33 s). To allow a precise readout of locomotor activity, the 
bioRxiv preprint doi: https://doi.org/10.1101/2021.12.23.474039; this version posted December 24,2021 . The copyright holder for this preprint (which was not certified by peer review) is the author/funder, who has granted bioRxiv a license to display the preprint in perpetuity. It is made available under aCC-BY-NC-ND 4.0 International license.

1083 mouse was placed on a spherical treadmill. Noxious pinch triggered coordinated calcium

1084 excitation in the central field of view regions corresponding to upper dorsal horn laminae. Scale

1085 bar, $100 \mu \mathrm{m}$.

1086 